\title{
WestVirginiaUniversity
}

THE RESEARCH REPOSITORY @ WVU

Graduate Theses, Dissertations, and Problem Reports

2013

\section{Human Action Recognition in Still Images}

Biyun Lai

West Virginia University

Follow this and additional works at: https://researchrepository.wvu.edu/etd

\section{Recommended Citation}

Lai, Biyun, "Human Action Recognition in Still Images" (2013). Graduate Theses, Dissertations, and Problem Reports. 670.

https://researchrepository.wvu.edu/etd/670

This Thesis is protected by copyright and/or related rights. It has been brought to you by the The Research Repository @ WVU with permission from the rights-holder(s). You are free to use this Thesis in any way that is permitted by the copyright and related rights legislation that applies to your use. For other uses you must obtain permission from the rights-holder(s) directly, unless additional rights are indicated by a Creative Commons license in the record and/ or on the work itself. This Thesis has been accepted for inclusion in WVU Graduate Theses, Dissertations, and Problem Reports collection by an authorized administrator of The Research Repository @ WVU. For more information, please contact researchrepository@mail.wvu.edu. 


\title{
Human Action Recognition in Still Images
}

\author{
Biyun Lai \\ Thesis submitted to the \\ College of Engineering and Mineral Resources \\ West Virginia University \\ in partial fulfillment of the requirement for the degree of \\ Master of Science in Computer Science
}

Guodong Guo, Ph.D., Chair

Xin Li, Ph.D.

Cun-Quan Zhang, Ph.D.

Lane Department of Computer Science and Electrical Engineering

\author{
Morgantown, West Virginia \\ 2013
}

Keywords: action recognition, image classification, supervised learning Copyright 2013 Biyun Lai 


\section{Abstract \\ Human Action Recognition in Still Images}

\section{Biyun Lai}

Recently still image-based human action recognition has become an active research topic in computer vision and pattern recognition. It focuses on identifying a person's action or behavior from a single image. Unlike the traditional action recognition approaches where videos or image sequences are used, a still image contains no temporal information for action characterization. It is more challenging to perform still image-based action recognition than the video-based, given the limited source of information as well as the cluttered background for images collected from the Internet.

Based on the emerging research in recent years, we dig into all possible cues from a single image: the whole image, human figure, action objects, and human-object interaction. We try to use supervised/semi supervised learning by using random forest/support vector machine. For the object labels, we also try automatic localization or manually label all the training/testing images. We have proved that besides the action scene and human action contour, action objects and their relations with centered human figure is important to still image human action recognition. 
The thesis is dedicated to my parents. They taught me that even the largest task can be accomplished if it is done one step at a time. 


\section{Acknowledgements}

First, I would like to express my most sincere thanks to my advisor, Dr Guodong Guo for his guidance, encouragement and help during my graduate years as a research assistant. What I learned from him will benefit all my life.

Second, I'd also like to thank my committee members: Dr Cun-Quan Zhang and Dr Xin Li and all my other friends in Morgantown West Virginia, for their help during the completion of my thesis.

Finally, I would like to thank the Lane Department of Computer Science and Electrical Engineering at West Virginia University for providing me with an great study environment during my years as a graduate student. 


\section{Contents}

1 Introduction 1

1.1 Motivation and Challenges . . . . . . . . . . . . . . . . 2

1.2 Research Timeline . . . . . . . . . . . . . . . . . . . . . . 4

2 Survey of Action Recognition in Still Images 5

2.1 Image Cues . . . . . . . . . . . . . . . . . . . . . 5

2.1 .1 Human Body . . . . . . . . . . . . . . . . . . . 6

2.1 .2 Body Parts . . . . . . . . . . . . . . . . . . 6

2.1 .3 Objects . . . . . . . . . . . . . . . . 7

2.1 .4 Human-Object Interaction . . . . . . . . . . . . . . . 8

2.1 .5 Context or Scene . . . . . . . . . . . . . . . . . . . 9

2.2 Methodologies . . . . . . . . . . . . . . . . . . . . 10

2.2 .1 Generative Models . . . . . . . . . . . . . . . . . . 10

2.2 .2 Discriminative Learning . . . . . . . . . . . . . . . 11

2.2.3 Learning Mid-level Features . . . . . . . . . . . . . . . . . . . . 11

2.2 .4 Multiple Features Fusion ～. . . . . . . . . . . . . . . . . 12

2.2 .5 Spatial Saliency . . . . . . . . . . . . . . . . . . . . 12

2.2.6 Conditional Random Field . . . . . . . . . . . . . . . . . . 13

2.2 .7 Pose Matching . . . . . . . . . . . . . . . . . . . . . . . . . 14

2.3 Relations to Other Topics . . . . . . . . . . . . . . . . . . . . 14

2.3.1 Video based Action Recognition . . . . . . . . . . . . . 15

2.3 .2 Object Recognition . . . . . . . . . . . . . . . . . . 15

2.3 .3 Pose Estimation . . . . . . . . . . . . . . . . . 16

2.3 .4 Scene Recognition . . . . . . . . . . . . . . . . . . 16

2.3.5 Image Retrieval . . . . . . . . . . . . . . . . . . . . 16 
$\begin{array}{llr}3 & \text { Databases } & 17\end{array}$

3.1 Database Introduction . . . . . . . . . . . . . . . . . . . 17

3.1.1 Sports Related Action Databases . . . . . . . . . . . . . . . 17

3.1 .2 Daily Activity Databases . . . . . . . . . . . . . . 20

3.1.3 Frames from Action Videos . . . . . . . . . . . . . . . . 22

3.1.4 Music Instruments Action Dataset . . . . . . . . . . . . . . . 23

3.2 Performance of Pascal VOC $2010 \ldots \ldots \ldots \ldots$

4 Action Learning $\quad 25$

4.1 Image Representation . . . . . . . . . . . . . . . . . . . . 25

4.1 .1 Whole Image . . . . . . . . . . . . . . . . 26

4.1 .2 Human Body . . . . . . . . . . . . . . . . . . . . 26

4.1 .3 Action Object . . . . . . . . . . . . . . . . 27

4.1 .4 Human-Object Interaction . . . . . . . . . . . . . . . . . . 28

4.2 Learning . . . . . . . . . . . . . . . . . . . . . . 31

4.2 .1 Support Vector Machine . . . . . . . . . . . . . . . . . 32

4.2 .2 Random Forest . . . . . . . . . . . . . . . . . . . . . . 32

4.3 Experiments . . . . . . . . . . . . . . . . . . . 32

$4.3 .1 \quad$ Overall Performance . . . . . . . . . . . . . . . . . 33

4.3 .2 Single Action Image Cues . . . . . . . . . . . . . . . . 33

4.3 .3 Object Localization . . . . . . . . . . . . . . . . . . . . . 34

5 Discussion $\quad 37$

5.1 Discussion . . . . . . . . . . . . . . . . . . 37

5.2 Concluding Remarks . . . . . . . . . . . . . . . . . . . 40

$\begin{array}{ll}\text { Bibliography } & 41\end{array}$ 


\section{1}

\section{Introduction}

Image classification has been an active research topic in computer vision and image processing, whose ultimate goal is to enable an automatic extraction of semantic information and knowledge from a single image or image sequences. Although various techniques have been developed for digital image processing, it is still a great challenge to enable machine automation, comparing with the human-level image understanding.

What we face here is a specific topic of image classification: human action recognition in still images. Is it possible for the computer to recognize what the human figure is doing in a single image? As far as we know, this is usually done in videos, with temporal information. It would be a difficult task. In this work, we mainly focus on recognizing the action of a bounded person in a still image belongs to which one of the possible candidate actions. We propose to delve further into all possible cues that an image can provide.

In Chapter 1, we reviewed the traditional video action recognition problem and talked about our motivation and challenges for this topic. We also provided a brief review of the publication in this research area, showing a popularity of this new trend.

In Chapter 2, we proposed a detailed review of the literature, mainly introducing and categorizing their methodologies.

We also introduced common databases in this research area in Chapter 3.

After we presented each human image by some feature descriptor and introduces our classifiers, we explained our methods in Chapter 4.

In Chapter 5, some discussions of further thoughts or suggestions for our works are presented. 


\subsection{Motivation and Challenges}

Recognizing human motion and action has been an active research topic in Computer Vision for more than two decades. This can also be indicated by a series of survey papers in the literature. Earlier review papers focused on human motion analysis and discussed human action recognition as a part, such as the surveys by Cedras and Shah

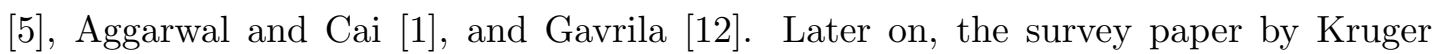
et al. 22$\rfloor$ classified human action recognition approaches based on the complexity of features to represent human actions and considered potential applications to robotics. The survey paper by Turaga et al. [38 $\rfloor$ covered human activity recognition with a categorization based on the complexity of activities and recognition methodologies. In Poppe's survey $\lfloor 31\rfloor$, various challenges in action recognition were addressed and novelties of different approaches were discussed. In Ji and Liu's survey [18], the concentration was on view-invariant representation for action recognition. They discussed related issues such as human detection, view-invariant pose representation and estimation, and behavior understanding. Finally, the most recent survey was given by Aggarwal and Ryoo in $\lfloor 2\rfloor$, which performed a comprehensive review of recognizing action, activity, gesture, human-objection interaction, and group activities. It discussed the limitations of many existing approaches and listed various databases for evaluations. The real-time applications were also mentioned.

Although motion-based / video-based human action recognition is still an active research topic in computer vision and pattern recognition, recent studies have started to explore action recognition in still images. As shown in Figure 1.1, many action categories can be depicted unambiguously in single images (without motion or video signal), and these actions can be understood well based on human perception. This evidence supports the development of computational algorithms for automated action analysis and recognition in still images. Considering the large number of single images distributed over the Internet, it is valuable to analyze human behaviors in those images. Actually, it has become an active research topic very recently $\lfloor 9\rfloor$.

An analogy to human (body-based) action recognition is facial expression recognition $\lfloor 10,49\rfloor$, sometimes called facial behavior understanding. In facial expression analysis, either single face images or face videos can be used. Different from action recognition, the studies of facial expressions using single images or videos are almost in 


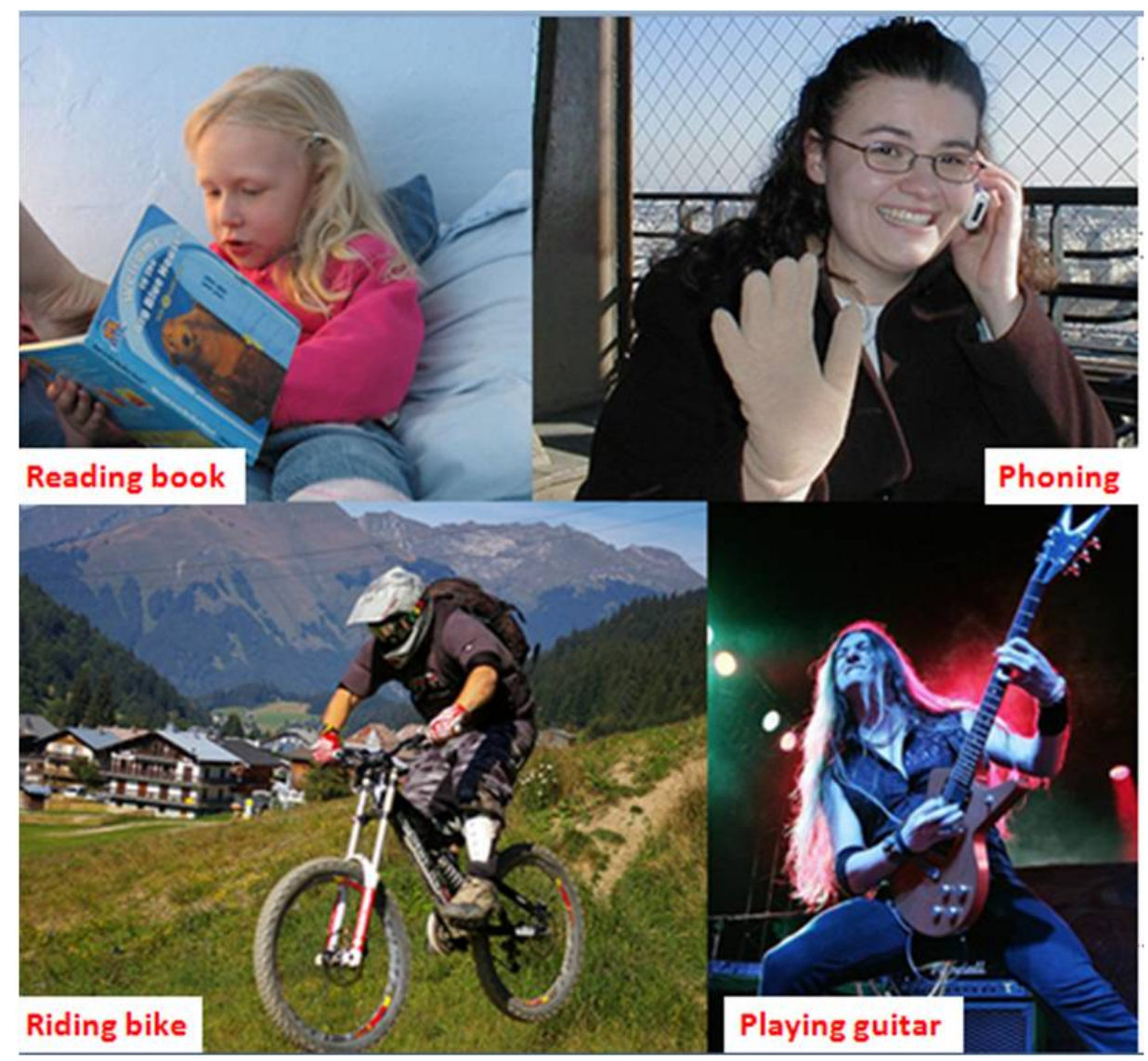

Figure 1.1: Some examples of still image-based action recognition. Only single images are sufficient to tell the corresponding actions, from Pascal VOC 2010 action datasets $\lfloor 9$.

parallel. The number of publications using either single images or videos are probably comparable in facial expression recognition.

Compared to the traditional video-based action recognition, still image-based action recognition has some special properties, which bring potential challenges. For example, there is no motion in a still image, and thus many spatiotemporal features and methods that were developed for traditional video-based action recognition are not applicable to still images. And also, it is not trivial to segment the humans from the background in still images $[8,19,32]$, since there is no motion cue to utilize and the scene can be very cluttered. 


\subsection{Research Timeline}

The study of still image-based action recognition has a very short history, comparing to the video-based action recognition research. Starting at about the year of 2006 [39], it appears to have some research papers on still image-based action recognition. Following 2006 , only a very small number of papers related to action recognition based on single images, since not many researchers have realized that it is an interesting topic. More papers were published recently. To show the trend of publications on this topic, an accumulative graph of the published papers in a yearly basis is drawn and shown in Figure 1.2. From the figure, we can see that there are more publications in very recent years, such as 2011 and 2012, with about 10 papers each year, while much less numbers before 2011. The research on this topic has become more active since 2011 .

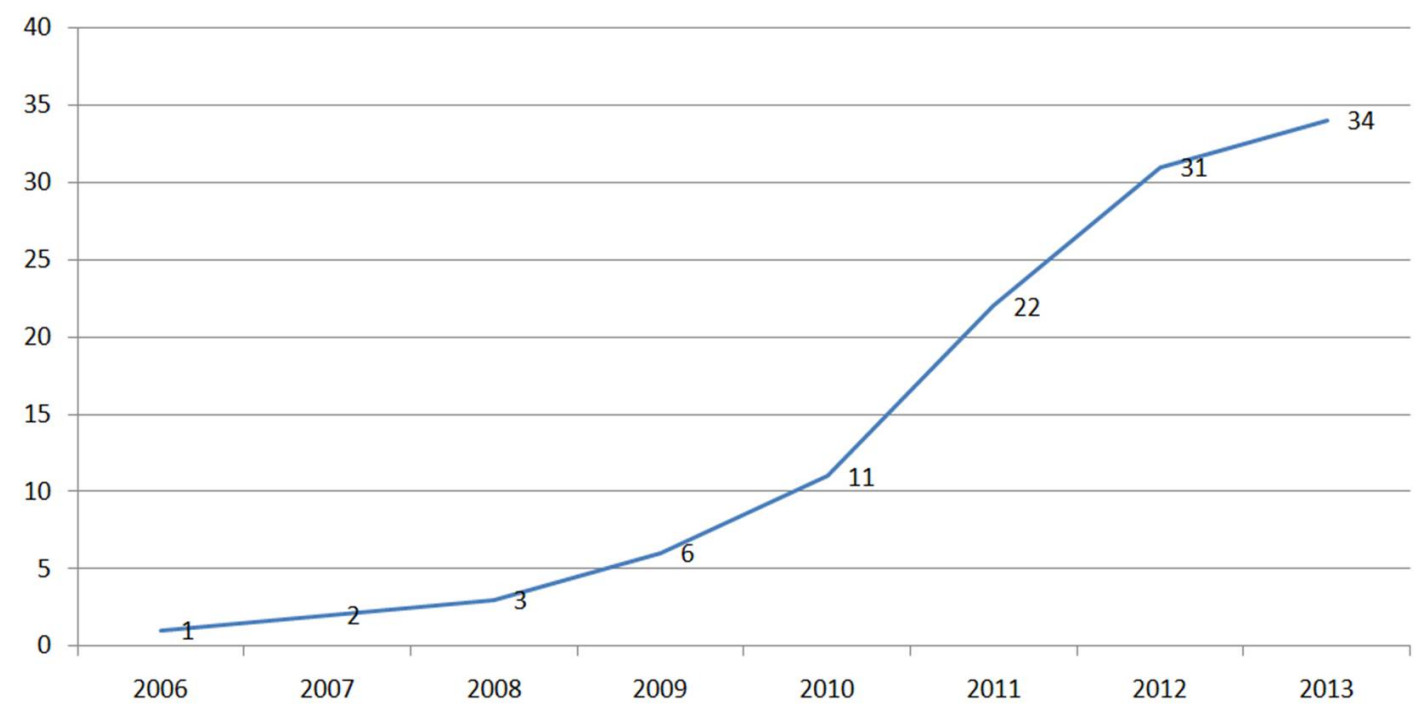

Figure 1.2: Timeline - An accumulative graph of the number of publications on still image-based action recognition. Based on the slope of the piecewise curve, one can see that there are more publications in 2011 and 2012 but less in earlier years. 


\section{Survey of Action Recognition in Still Images}

From previous chapter, we already know that human action recognition in still images has been a very hot topic in computer vision areas. This is the right time for us to give a review of the past breakthroughs, giving inspirations for our current works and future development. We have organized the reviewed publications in several ways:

Section 1 introduces those methodologies in an image representation way, exploring how many image cue they have used. Here, an image cue refers to a high level concept, which may be a substantial human figure, or a relationship between human and the involved action objects.

In section 2, we explain the existing methodologies in several categories by how they learn the action recognition.

Finally, we would talk about how research in still image based action recognition related to other topics in computer vision.

\subsection{Image Cues}

We define here an image cue refers to a high level concept, in whatever way they are recognized in advance. The most popular high-level cues for still image-based action recognition include: the human body, body parts, action-related objects, human object interaction, and the whole scene or context. These cues can characterize human actions from different aspects. Some approaches employed more cues, while some others used 
less. How to explore cues efficiently help improve the performance of recognizing the human action.

\subsubsection{Human Body}

Human body is an important cue for still image-based action recognition. Most of the existing approaches use the human body cue for action representation. The human body can be detected automatically $[8,19\rfloor$ in images or manually labeled $[9\rfloor$. Usually the bounding box of the human is used to indicate the location of the person and determine the image region for human body information extraction.

There are approaches 17 . that extract features in areas within or surrounding the human bounding boxes. They defined a person setting in each image with $1.5 \times$ the size of the human bounding box, and resized each cropped region into a new size such that the larger dimension is 300 pixels. These regions are then represented using some low-level features.

On the other hand, some critical patches can be found within the bounding box of human body. For instance, Yao et al. [48 . used the random forest method with some variations to search the useful, discriminative patches from the human body region for action recognition.

Semantic features such as the attributes can also be used to describe the actions in images with the human body [46]. A global representation of the attributes and binary (yes/no) classifiers can be used to learn each attribute for action analysis. The attributes are defined as linguistically related descriptions of human actions. Most of the attributes used were related to verbs in human language. For instance, the attributes of "riding" and "sitting (on a bike seat)" can be used to describe the action of "riding a bike." And also, one attribute may be shared by different actions. For instance, "riding" can be shared by "riding a bike" and "riding a horse." However, there are different attributes between any two actions so that the actions can be differentiated using the attributes.

\subsubsection{Body Parts}

Rather than the whole human body, body parts can be more related to action execution. When performing different actions, e.g., throwing a ball or using a computer, the body 
parts, e.g., the arms, can be in different locations or with different poses. Based on this, the cue of body parts may be used for action characterization.

\section{Variable nodes}

- Image-independent factors

․ Image-dependent factors

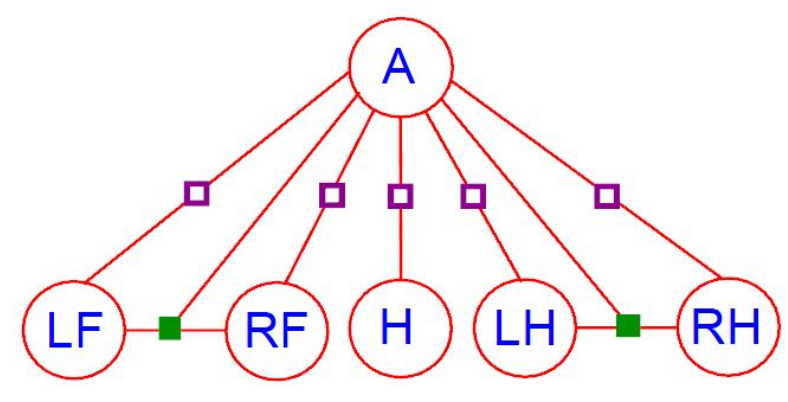

Figure 2.1: The graphical model - Originally shown in $\mid 33$. The model of a person contains six variable nodes encoding the positions of five body parts and the action label. The considered parts $\mathrm{p}=\{H, R H, L H, R F, L F\}$ correspond to head, right-hand, left-hand, right-foot and left-foot, respectively, as well as $\mathrm{K}$ action classes $\mathrm{A}$. The links between the nodes encode action dependent constraints on the relative position of body parts and their appearance.

A graph is usually a good model to represent the connections and relations between different body parts. Raja et al. [33. considered a graphical model containing 6 nodes, encoding positions of 5 body parts and the action label. See Figure 2.1 for an illustration of their work. Body parts $\mathrm{p}=\{H, R H, L H, R F, L F\}$ correspond to head, right-hand, left-hand, right-foot and left-foot, respectively, as well as the action class label node A. The links between the nodes encode action-dependent constraints on the relative positions of body parts and their appearance. Using the body part detection results, relations between positions of hands and feet in images are also modeled and interpreted as being proportional to the joint probability of the right- and left-part location for a given action.

\subsubsection{Objects}

When performing actions by humans, there are objects related to the actions. This can be observed in many still images of human actions. Thus it is natural to consider the 
related objects for human action characterization. Different actions might be related to different objects. By knowing the related objects, it can help to recognize the corresponding actions. For example, a horse (with a human) is possibly related to the action of "riding a horse," or a phone (with a person) could be related to the action of "phoning." Researchers have realized the importance of using object information to help action recognition in still images.

Prest et al. $[32\rfloor$ used the results from objectness $[3\rfloor$ to calculate the probability of a certain patch being an object. The objectness method can find multiple candidates of objects that can be related to actions regardless of the actual classes of the objects, such as bike, horse, phone, etc.

\subsubsection{Human-Object Interaction}

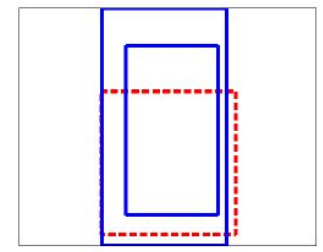

motorbike

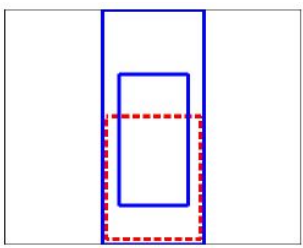

bicycle

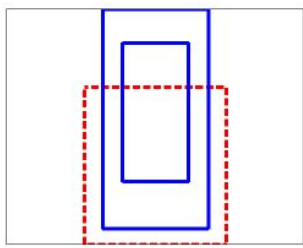

horse

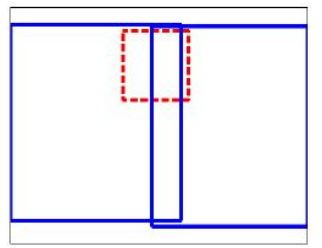

tvmonitor

Figure 2.2: A spatial model of object person interaction. Each one shows the modes of the bounding boxes of the person (blue) relative to the bounding box of the object (red), originally shown in 29$]$

In addition to the co-occurrence of humans and objects and modeling of them separately, the interaction between humans and objects is also useful for action recognition in still images. For instance, the relative position between a person and the actionrelated object (e.g., a book for reading), and the relative angle between the person and object (e.g., the person is above the bike when he/she is riding a bike), the relative size of the person and object (e.g., a phone (in calling) is much smaller than a horse (in riding) in the two different actions), etc. The configuration of humans and objects in executing actions have been pursued by several researchers.

Maji et al. [29」 learned a mixture model of the relative spatial locations between the person's bounding box and the object's bounding box in still images, as shown in Figure 2.2. For each object type, they fit a two component mixture model of the 
predicted bounding box to model various relative locations between the person and object.

\subsubsection{Context or Scene}

The background in an image usually refers to the image region with the foreground human and/or object removed. It may be taken as the context or scene of an executed action. In some cases, the whole image could be considered as the context or scene for action analysis, especially when the foreground (e.g., human and object) occupies a relatively small area in the still image. In reality, some actions are performed in specific scenes, e.g., swimming in water, driving on the road, etc. So extracting information from the action context or the whole scene can be helpful for still image-based action analysis and recognition.

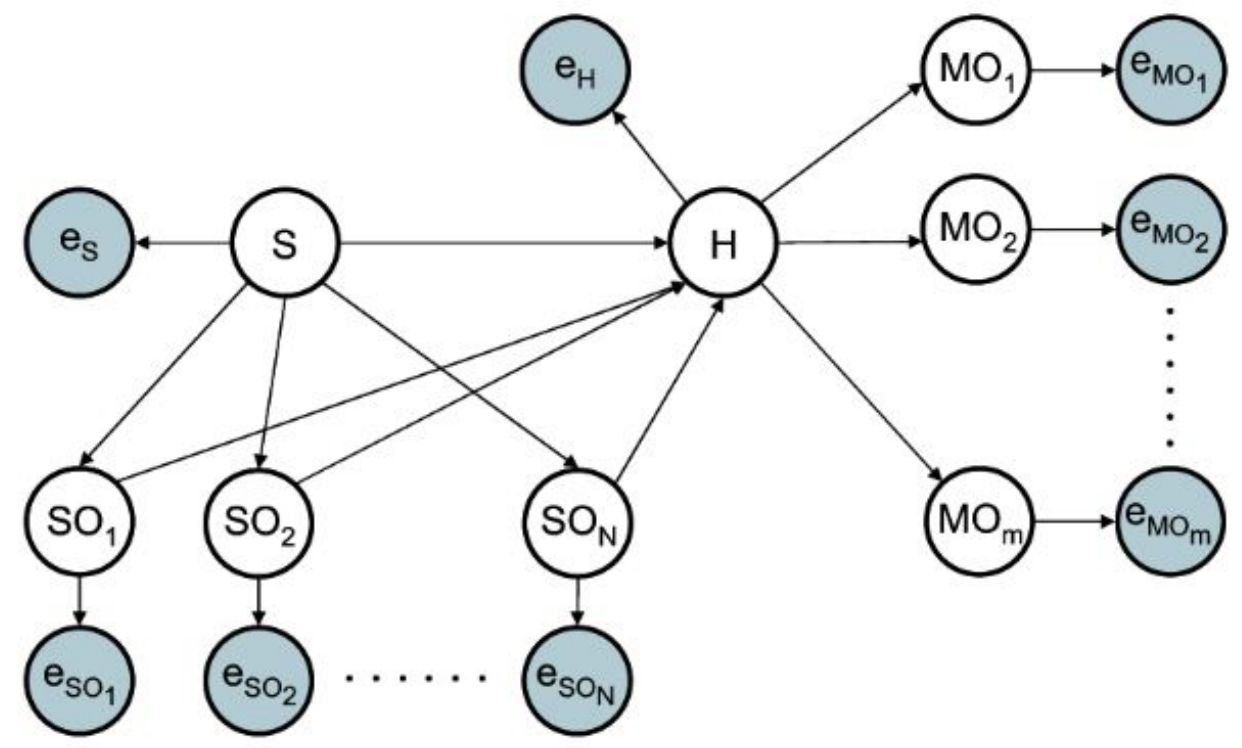

Figure 2.3: The model consists of four types of nodes, corresponding to scene/event(S), scene objects(SO), manipulable objects(MO), and human(H). The observed (evidence) and hidden nodes are shown in blue and white, originally shown in $\lfloor 14$.

Gupta et al. $\lfloor 14\rfloor$ encoded the scene for action image analysis. Their Bayesian model consists of four types of nodes, corresponding to the scene/event (S), scene objects (SO), manipulable objects (MO), and human $(\mathrm{H})$. See Figure 2.3 for an illustration of the approach. 
Some other approaches used the background information based on whole image classification. The extracted feature or resulted confidence measure from the background may be fused with other results at either the feature- or decision-level. For example, Prest et al. $[32\rfloor$ extracted features from the whole image with GIST for action image analysis.

Although the action context or scene is useful for action recognition, the image background may have negative effects on action analysis, especially when the background is too noisy and cluttered. Furthermore, different actions might be performed in the same or similar scene in which the context or scene may not provide helpful information to separate those actions.

\subsection{Methodologies}

Given various image representations, either high-level cues or just low-level feature extractions, the next step is to learn the actions from training examples. The learned models or classifiers can then be used to recognize actions from the unseen, test images. Different learning methods have been proposed by researchers. We categorize the action learning methods into different categories, such as general models, discriminative learning, learning mid-level features, fusing multiple features, extracting spatial saliency, conditional random field, and pose matching. We will introduce various action learning methods under the seven categories in this section.

\subsubsection{Generative Models}

Generative models usually learn the statistical distributions for action classes, which can randomly generate the observable data. Some see in an image isolated scene or objects are all isolated and their independent occurrences are respectively found to be a clue to recognize the action $\lfloor 26\rfloor$. Others may develop a generative model, where the images usually present human as the action center, interacting with object and scene to some degree $[14\rfloor$.

A Bayesian model was used in Gupta et al. [14. See Figure 2.3 for an illustration. A fully supervised approach was taken to train the Bayesian model for image interpretation. The parameters were learned for individual likelihood functions and the conditional probabilities which model the interactions between the object and scene. 
To learn parameters of individual likelihood functions, they trained individual detectors separately. Given the evidential variables or the observations, the goal is to estimate scene variable, scene objects, human, manipulable objects, and inference over the graphical model.

\subsubsection{Discriminative Learning}

Discriminative learning is appropriate for distinguishing different action classes, without turning to learning the complex generative models. Among many classifiers, SVM (Support Vector Machine) [34」 is the most popular. A one-vs-all or several binary SVM classifiers were trained for action classification. Some others such as Komiusz and Mikolajczyket $[20,21\rfloor$ used the Kernel Discriminant Analysis (KDA) classifier with $\chi^{2}$ kernel. What they believe is these learning classifiers are enough to accomplish these recognition tasks as a classification problem, while differences among methodologies would be their input features or learned image cue presentation.

\subsubsection{Learning Mid-level Features}

Different from low-level features such as the SIFT [28」, HOG $\mid 6\rfloor$, etc., some middle level features can be learned from the action images. Most of them are based on the extracted low-level features.

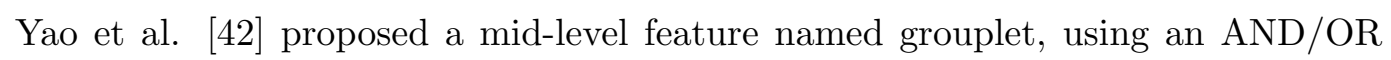
structure on low-level features, which are computed from the SIFT codebook over the dense grid. The feature unit, denoted by $(A, x, \sigma)$, indicates that a codeword of visual appearance $\mathrm{A}$ is observed in the neighborhood of location $\mathrm{x}$ (relative to a reference point). The spatial extent of $\mathrm{A}$ in the neighborhood of $\mathrm{x}$ is expressed as a 2D Gaussian distribution $\mathrm{N}(\mathrm{x}, \sigma)$. Each feature unit captures a specific appearance, location, and spatial extent information of an image patch.

Maji et al. $\lfloor 29\rfloor$ learned 1200 action-specific poselets. Based on the assumption that if a pose is discriminative, there will be many examples of that poselet from the same action class, they measured the discriminativeness by the number of within class examples of the seed windows in the top $\mathrm{k}$ nearest examples to the poselet. The representative poselets for each action class are trained using HOG-based features and the SVM classifiers. 


\subsubsection{Multiple Features Fusion}

Multiple features can be extracted to help improve the action recognition accuracy, in feature level (e.g., histogram concatenation, etc.) or score level. The assumption in fusion-based approaches is that multiple features may complement each other and a combination of them may characterize the actions better than each single feature. Thus the fusions of multiple features are expected to improve the action recognition accuracies. Some fusion methods were carefully investigated in $\lfloor 19\rfloor$ for different features, incorporating shape and color information.

Prest et al. $\mid 32\rfloor$ extracted three descriptors: human-object interaction, whole image, and human pose cues. They used a separate RBF kernel for each descriptor and computed a linear combination of them. A multi-kernel one-vs-all SVM classifier was learned.

In $\lfloor 19\rfloor$, Khan et al. exploited the efficiency of color in still image recognition. They examined various color descriptors for representing action images. Then they tried different fusions of shape and color features for both action classification and detection.

\subsubsection{Spatial Saliency}

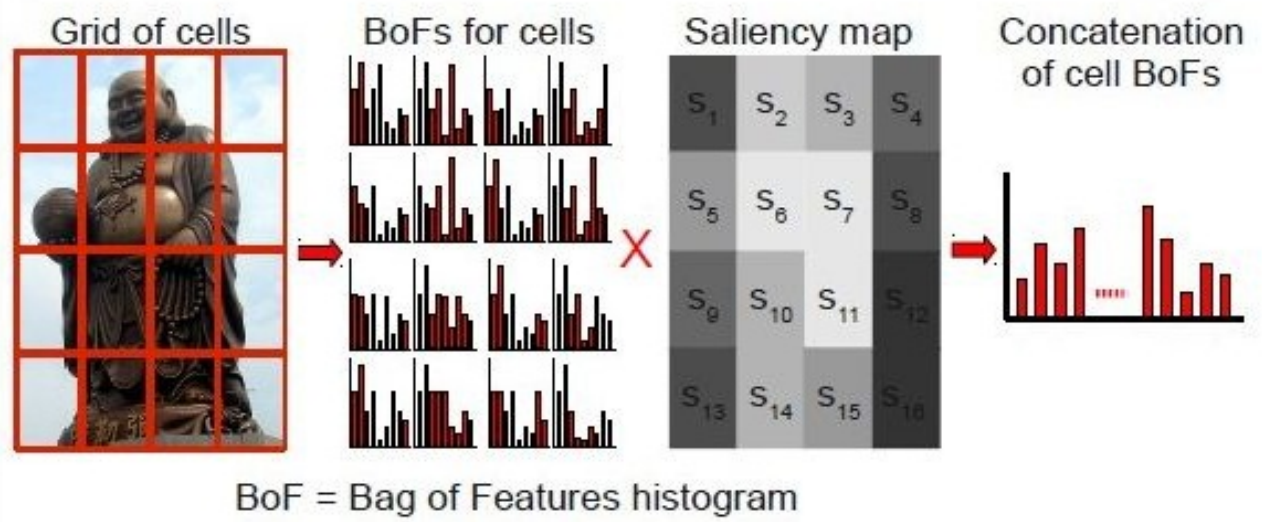

Figure 2.4: originally shown in 36. The images are represented by concatenation of cell bag-of-features weighted by the image saliency maps. A block coordinate descent algorithm is used to learn the model. Using a latent SVM, they optimized iteratively the hyperplane vector $\mathrm{w}$ while keeping the saliency maps of the positive images fixed, and optimized the saliency while keeping w fixed. 
Sharma et al. [36] defined image saliency as a mapping $s: G \rightarrow R$, where $\mathrm{G}$ is a spatial pyramid like uniform grid $\lfloor 24\rfloor$ of image, $c \in G$ is a region of the image, and $\mathrm{s}(\mathrm{c})$ gives the saliency of the region. They proposed a model consisting of three components: (i) the separating hyperplane $\mathrm{w}$, (ii) the image saliency maps $s^{i}$, and (iii) a generic saliency map $\bar{s}$ to regularize the image saliency maps. The saliency map of an image maximizes the classification score while penalizes the deviation from the generic saliency map. See Figure 2.4 for some illustrations. The images were represented by concatenation of cells of bag-of-features, weighted by the image saliency maps. Using a latent SVM, they optimized iteratively the hyperplane vector $\mathrm{w}$ while keeping the saliency maps of the positive images fixed, and the saliency map while keeping $\mathrm{w}$ fixed.

\subsubsection{Conditional Random Field}

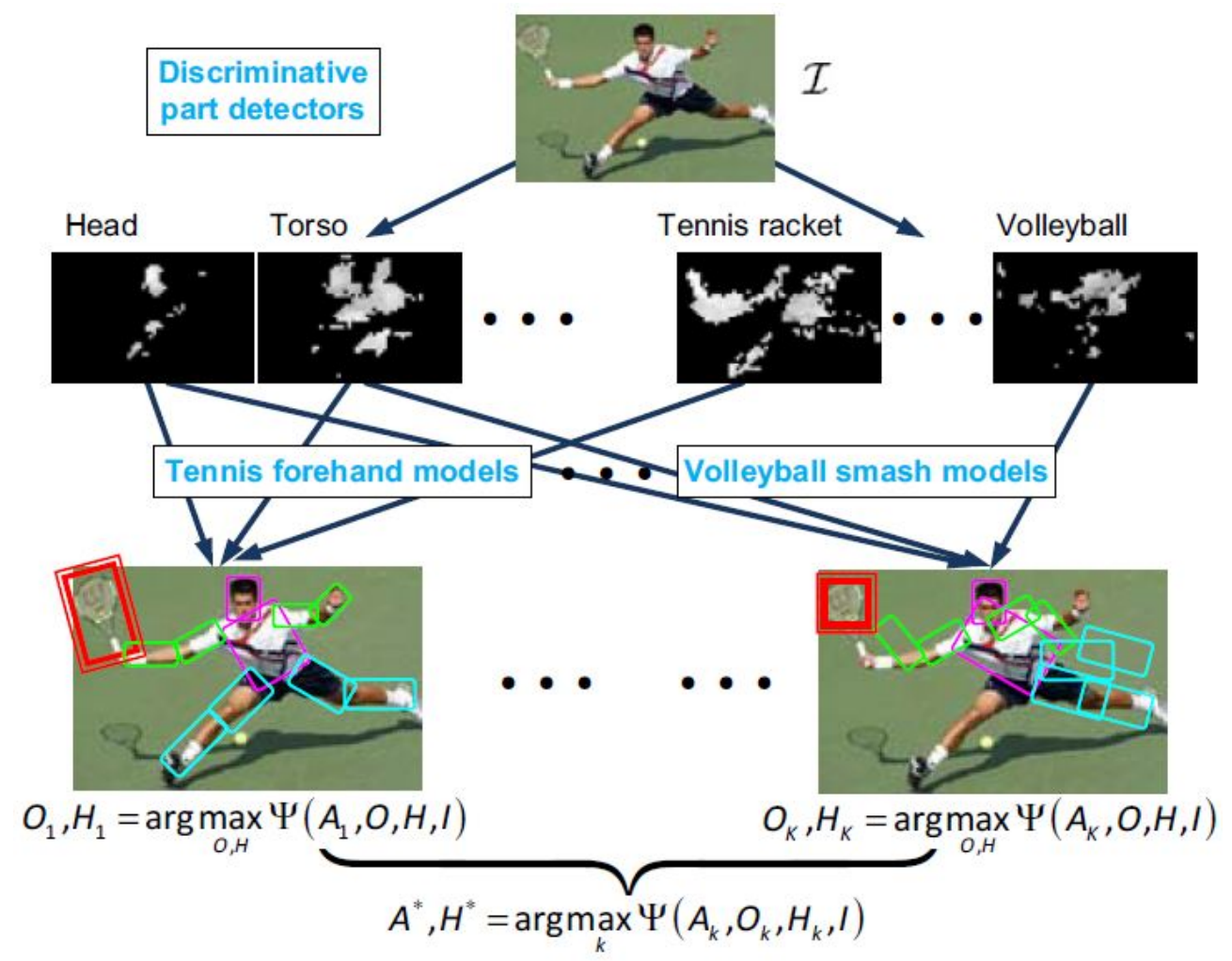

Figure 2.5: The framework of the inference method, originally shown in 43]. Given an input image I, the inference results are: (1) object detection results $O_{k}$ (e.g., $O_{1}$ is the tennis racket detection result); (2) human pose estimation result $H^{*}$; (3) activity classification result $A^{*}$. 
Yao and Fei-Fei $[43\rfloor$ used a conditional random field (CRF) model for action analysis. As illustrated in Figure 2.5, the inference procedure is the following: To detect the tennis racket in the image, the likelihood of the image is maximized, given the models learned for tennis-forehand. This is achieved by finding a best configuration of human body parts and the object (tennis racket) in the image, which is denoted as $\max _{O, H} \Psi\left(A_{k}, O, H, I\right)$ in the figure where $\mathrm{A}$ indicates action, $\mathrm{H}$ is the pose, $\mathrm{O}$ is the object, and $\mathrm{I}$ is the image. In order to estimate the human pose, they computed $\max _{O, H} \Psi\left(A_{k}, O, H, I\right)$ for each activity class and find the class $A^{*}$ that corresponds to the maximum likelihood score. This score can be used to measure the confidence of activity classification as well as human pose estimation.

Later, Yao and Fei-Fei $\lfloor 45,47\rfloor$ extended their model by introducing a set of atomic poses. They learned an overall relationship between different activities, objects, and human poses, rather than modeling the human-object interactions for each activity separately as in [43]. Instead of limiting to one human and one object interaction [43], the extended model can deal with the human interactions with any number of objects. The new model incorporates a discriminative action classification component and uses the state-of-the-art object and body part detectors, which further improves the recognition accuracy.

\subsubsection{Pose Matching}

Some approaches $[39\rfloor$ to action recognition are mainly based on matching human body poses. The matching scheme is especially to exploit body shape and pose information. From a sketch of human body poses, it was assumed that there is a great similarity among intra-class poses and the matching of poses can recognize actions. To deal with variations of intra-class poses, multiple poses can be used to represent a certain class.

\subsection{Relations to Other Topics}

Still image-based action recognition is not an isolated topic. It is closely related to some other research problems, such as video based action recognition, object recognition, scene recognition, image retrieval and pose estimation. See Figure 2.6 for a visualization of the relations. The progress in those related problems may help to enhance action recognition in still images. On the other hand, action recognition can help 


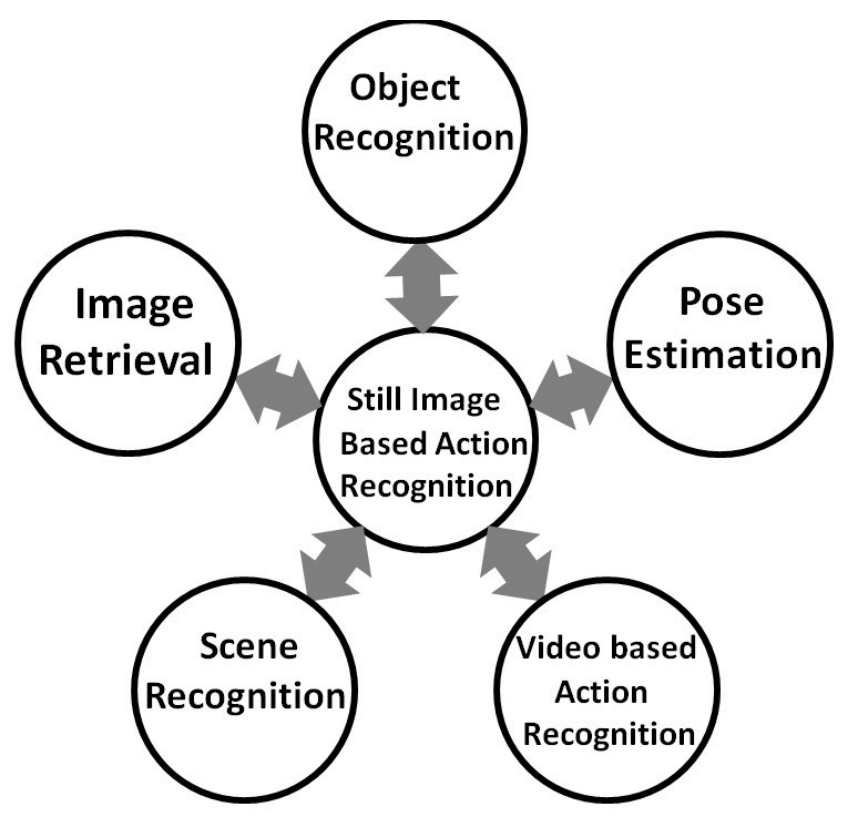

Figure 2.6: Other topics related to still image-based action recognition.

other research problems by providing refined semantic meaning in images, e.g., what actions are performed in the still images to be annotated.

\subsubsection{Video based Action Recognition}

Ignoring the motion cue, video based action recognition could be done by analyzing individual frames, which also applies to image-based action recognition. Thurau et al. [37] got training data from video frames and their method was applied to video-based action recognition on the KTH dataset $[35\rfloor$.

The approaches to image-based action recognition could be used directly or adaptively for video-based action recognition $\lfloor 14,41$. The model could be trained directly using the image data. Ikizler et al. $\lfloor 16,17\rfloor$ used images collected from the Web to learn image representations of actions, which could also benefit the automated annotation of actions in videos.

\subsubsection{Object Recognition}

The grouplet $\lfloor 42\rfloor$ can be used to find subtle differences in similar scenario such as whether the human is playing or just holding a music instrument. Since it is also a 
general framework to mine structured visual features in images, grouplet also has better performance of object recognition on Caltech101 dataset [11]. Similarly, their method [48] on seeking discriminative patches in action images may also serve the purpose of finding subtle differences of all kinds of birds on a subordinate object categorization dataset of 200 bird species [40].

Yao et al. $[43,45,47\rfloor$ also detected the presence and location of the object given an action with Human-Object Interaction, estimate pose, and recognize the action by learning one model for each module.

Some other works $\lfloor 20,21,23\rfloor$ also proved that the enhancement in the classifier level could apply in most popular object datasets. Integrating spatial information and improving the soft assignment of visual words $[20,21\rfloor$ can also help to improve the object recognition performance.

\subsubsection{Pose Estimation}

Similar to object detection, Yao et al.[43, 45, 47] showed that human pose estimation can be significantly improved by the detected object context.

Approaches in $18,29,32,33$. emphasized the efficiency of poses for action recognition. This is very intuitive. In action images, human are usually the focus points, with various articulated body poses. To determine the poses, prior knowledge of action classes may help to reduce the number of candidate poses to a few.

\subsubsection{Scene Recognition}

Sharma et al. [36. also performed action recognition in the scene dataset $\lfloor 24\rfloor$, which contains 15 scene categories, e.g., beach, office, etc. The task is a multi-class classification problem. They showed that in both scene analysis and action recognition, extracting discriminative image patches are critical to determine the class labels. Action recognition may help scene recognition.

\subsubsection{Image Retrieval}

In $\mathrm{Li}$ et al.'11 [27,, action recognition is conducted on the retrieved web images to determine whether they are the desired results from text keyword searching. It can help to achieve a better performance on image retrieval. 


\section{3}

\section{Databases}

Following last chapter, we present more statistics and information here for the research of human action recognition in still images. In this chapter, we would like to give a relatively detailed introduction of all common databases used in all researches for this topic, including a performance review of some databases with a high popularity. We will also emphasize the databases we used in our experiments, which are Pascal VOC 2010 and 2011 datasets $[9\rfloor$.

\subsection{Database Introduction}

There are many public datasets available to validate different methods for action recognition in still images. We present the widely used datasets and categorize them into different categories. The statistics of all datasets are shown in Table 3.1 with other information as well, e.g., the source of the datasets, and which papers conducted experiments on each dataset.

\subsubsection{Sports Related Action Databases}

In collecting action databases, action images in sports are the earliest and of the most popular usage for recognition, probably due to the relatively small human pose variations within the same actions in sports activity, and the distinctiveness and uniqueness of specific sports actions in single images. 
Table 3.1: Databases assembled for still image-based action recognition.

\begin{tabular}{|l|l|l|l|}
\hline Dataset & \# of Images & \# of Classes & Source \\
\hline The Sports Dataset $\lfloor 14\rfloor$ & 300 in total & 6 & Internet \\
\hline *Skating dataset $[39\rfloor$ & 1400 in total & 10 & Videos \\
\hline *Baseball dataset $\lfloor 39\rfloor$ & 4500 in total & 7 & Sports news \\
\hline *Basketball dataset $\lfloor 39\rfloor$ & 8500 in total & 8 & Sports news \\
\hline *Sports Events $\lfloor 26\rfloor$ & 137 to 250 per class & 8 & Internet \\
\hline Pascal VOC $2010\lfloor 9\rfloor$ & 50 to 100 per class & 9 & Internet \\
\hline Pascal VOC $2011\lfloor 9\rfloor$ & 200 or more per class & 10 & Internet \\
\hline Pascal VOC $2012\lfloor 9\rfloor$ & 400 or more per class & 10 & Internet \\
\hline Stanford 40 Actions $\lfloor 46\rfloor$ & 180 to 300 per class & 40 & Internet \\
\hline Willow Dataset \lfloor\rfloor & 968 in total & 7 & Internet \\
\hline 89 Action Dataset $\lfloor 25\rfloor$ & 2038 in total & 89 & Pascal 2012 \\
\hline *Action Images by Ikizler $\lfloor 15\rfloor$ & 467 in total & 6 & Internet \\
\hline *Retrieved Web Images $\lfloor 16\rfloor$ & 2458 in total & 5 & Internet \\
\hline *Action Images by Li $\lfloor 27\rfloor$ & 400 per class & 6 & Internet \\
\hline TBH $\lfloor 32\rfloor$ & 341 in total & 3 & Google Images \\
\hline Weizmann dataset $\lfloor 37\rfloor$ & - & 10 & Videos \\
\hline KTH dataset $[33\rfloor$ & 789 in total & 6 & Videos \\
\hline PPMI $\lfloor 42\rfloor$ & 300 per class & 7 & Internet \\
\hline
\end{tabular}

* dataset names are given by us. 


\subsubsection{The Sports Dataset}

The sports dataset first used in $\lfloor 14\rfloor$ has six actions: tennis-forehand, tennis-serve, volleyball-smash, cricket-defensive shot, cricket-bowling and croquet-shot. The authors illustrated that the changes between actions are mainly on poses rather than the object or scene. Each action class contains 20 to 30 training images and testing images, respectively. The classes were selected so that there are significant confusions due to the same scene and similar poses. For example, the poses during volleyball-smash and tennis-serve are quite similar and the scenes in tennis-forehand and tennis-serve are exactly the same. This is a widely used dataset for human action recognition involving objects.

\subsubsection{Skating Dataset}

Wang et al. [39 $\rfloor$ assembled three datasets in 2006. The first dataset is a collection of images from six videos of different figure skaters. These videos were automatically filtered. Frames with complicated backgrounds (consisting of a large number of edges) were removed, resulting in a simplified set of 1400 images. The figure skating clusters were given the following 10 labels: face close-up picture, skates with arms down, skates with one arm out, skater leans to his right, skates with both arms out, skates on one leg, sit spin leg to left of the image, sit spin leg to right, camel spin leg to left, and camel spin leg to right.

\subsubsection{Baseball Dataset}

Wang et al. [39」 collected the baseball clusters consisting of 4500 images, which were collected by querying the captions of sports news photos for professional sports team names. These datasets are significantly more challenging, containing substantial background clutter, and a wide range of content. The baseball clusters have 7 labels: face close-up picture, right-handed pitcher throws, right-handed pitcher cocks his arm to throw, runner slides into base, team celebrates, batter swings, batter finished swinging.

\subsubsection{Basketball Dataset}

The basketball clusters were also collected by Wang et al. [39] with 8500 images. There are 8 labels: a player goes for a lay-up above the defenders, a player goes for a lay-up 
against a defender, a player goes for a jumpshot while another one tries to block, a player goes for a lay-up leaning to his right, a player drives past another, a player has his shot blocked, a player leaps by his defender for a shot, and a player posts up.

\subsubsection{Sports Events Dataset}

Li et al. 27$\rfloor$ compiled a dataset, containing 8 sports event categories collected from the Internet: bocce, croquet, polo, rowing, snowboarding, badminton, sailing, and 5 rock climbing. The number of images in each category varies from 137 (bocce) to 250 (rowing). They had also obtained a thorough ground truth annotation for every image in the dataset. This annotation provides information for event class, background scene class (es), most discernable object classes, and detailed segmentation of each objects. For each event class in their experiments, 70 randomly selected images were used for training and 60 for testing.

\subsubsection{Daily Activity Databases}

Datasets in this category contain common activities performed by humans in daily lives, which have less controversy than other categories of databases.

\subsubsection{Pascal VOC action datasets}

In our experiments, we also use Pascal VOC 2010 and 2011 datasets due to their popularity. Pascal VOC competition includes still image-based action recognition starting from $2010\lfloor 9\rfloor$. There are 9 actions: phoning, playing a musical instrument, reading, riding a bicycle or motorcycle, riding a horse, running, taking a photograph, using a computer, or walking. Only subset of people are annotated (bounding box of the human + action). All people in dataset are labelled with exactly one action class.

Later in 2011, this dataset was extended about 5 times larger in size, and one more action called jumping was added to the original 2010 dataset. There is a minimum of around 200 people per action category. Actions are not mutually-exclusive, which means there could be one person with more than one action labels in the same image. Besides these changes, training and test images belonging to 'other' action class were collected in the dataset, increasing the difficulty in action analysis. 
In 2012, the dataset was expanded again. About $90 \%$ increase in size over VOC 2011. There is a minimum of around 400 people per action category. A single point located somewhere in the human body was also annotated for each image.

\subsubsection{Stanford 40 Actions Dataset}

Yao et al. [46」 collected a challenging, large scale dataset, called Stanford 40 Actions, containing 40 diverse daily human actions, such as brushing teeth, cleaning the floor, reading book, throwing a frisbee, etc. All images were obtained from Google, Bing, and Flickr. 180 to 300 images were collected for each class. There are 9352 images in total. They provided bounding boxes for the humans who are doing one of the 40 actions in each image. The authors randomly selected 100 images in each class for training, and the remaining for testing.

\subsubsection{Willow Dataset}

Delaitre $[7\rfloor$ collected the willow action dataset from original consumer photographs, depicting seven common human actions: interacting with computers, photographing, playing a musical instrument, riding bike, riding horse, running and walking. Images for the riding bike action were taken from the Pascal 2007 VOC Challenge and the remaining images were collected from Flickr by querying on keywords such as running people or playing piano, resulting in a total of 968 photographs with at least 108 images for each class. They split the dataset into two parts: 70 images per class for training and the remaining for testing. Each image was manually annotated with bounding boxes indicating the locations of people.

\subsubsection{The 89 Action Dataset}

Le et al. $\lfloor 25\rfloor$ assembled a dataset from 11.5 thousand images of the PASCAL 2012 VOC trainval set $[9\rfloor$, selecting all those images representing a human action, resulting in 2,038 images. They manually annotated these images with a verb to obtain the label of the human action (verb-object). The data set was annotated with 19 objects and 36 verbs, which are combined to form 89 actions. Similar to the training vs. validation split used in the PASCAL competition, their human action data set consists of 1,104 images for training and 934 images for validation. 


\subsubsection{Action Images by Ikizler}

Ikizler et al. $\lfloor 15\rfloor$ built a dataset from various sources like Google Image Search, Flickr, BBC Motion database, etc. This dataset consists of 467 images and includes 6 different actions: running, walking, catching, throwing, crouching and kicking.

\subsubsection{Retrieved Web Images}

Ikizler et al. [16] retrieved images from the web. Part of their works were done on refining the initial results of keyword queries to an image search engine. In the end, there are 2458 images in total in the dataset, containing 384 running, 307 walking, 313 sitting, 162 playing golf, and 561 dancing images.

\subsubsection{Action Images by $\mathrm{Li}$}

Li et al. [27」 collected about 2400 images for six action queries: phoning, playing guitar, riding bike, riding horse, running and shooting. Most of the images were collected from Google Image, Bing and Flickr, and others are from PASCAL VOC 2010 [9. Each action class contains about 400 images.

\subsubsection{TBH Dataset}

Prest et al. $\lfloor 32\rfloor$ introduced an action dataset called TBH. It was built from Google Images and the IAPR TC-12 data set, containing three actions: playing trumpet, riding bike,and wearing hat. Split 100 positive images into training (60) and testing (40) for playing trumpet class. For the actions of riding bike and wearing hat, images from the IAPR TC-12 dataset were used. The dataset contains 117 images for riding bike (70 training, 47 testing) and 124 images for wearing hat (74 training, 50 testing). Images were only annotated with the action class labels.

\subsubsection{Frames from Action Videos}

Still images may also be extracted from some action videos. The extracted image frames usually have a relative static or cleaner background. 


\subsubsection{Weizmann dataset}

Thurau et al. [37 J used still images extracted from the popular Weizmann action videos [13]. The dataset contains 10 different actions: bend, jack, side, skip, run, pjump, jump, walk, wave1, and wave2, performed by 9 subjects.

\subsubsection{KTH dataset}

Raja et al. [33」 executed human pose estimation and action recognition in still image frames extracted from the KTH dataset [35]. The dataset contains images of 6 classes: boxing, handclapping, hand-waving, jogging, running and walking. The training and test sets were separated by the identities, containing 461 and 328 cropped images, respectively.

\subsubsection{Music Instruments Action Dataset}

\subsubsection{People-playing-musical-instruments}

Yao and Fei-Fei $\lfloor 42\rfloor$ assembled a dataset called People-playing-musical-instruments (PPMI). The PPMI consists of 7 different musical instruments: bassoon, erhu, flute, French horn, guitar, saxophone, and violin. Each class includes 150 PPMI+ images (humans playing instruments) and 150 PPMI- images (humans holding the instruments without playing).

\subsection{Performance of Pascal VOC 2010}

To understand the current status of still image-based action recognition, we present the action recognition accuracies obtained in previous approaches. We select to present the reported recognition results on the Pascal VOC 2010 Action Dataset [9]. The recognition accuracies of different methods on are shown in Figures 3.1. VOC datasets set up a new measurement of mean Average Precision (mAP), which is a calculation of the area under the precision-recall curve. This is different from the traditional accuracy measure, somehow becoming a standard for performance evaluation in action recognition.

We can observe that the recognition accuracies are in the range from about $59 \%$ to $68 \%$ on Pascal VOC 2010 Action Dataset. The accuracies are improving with the 


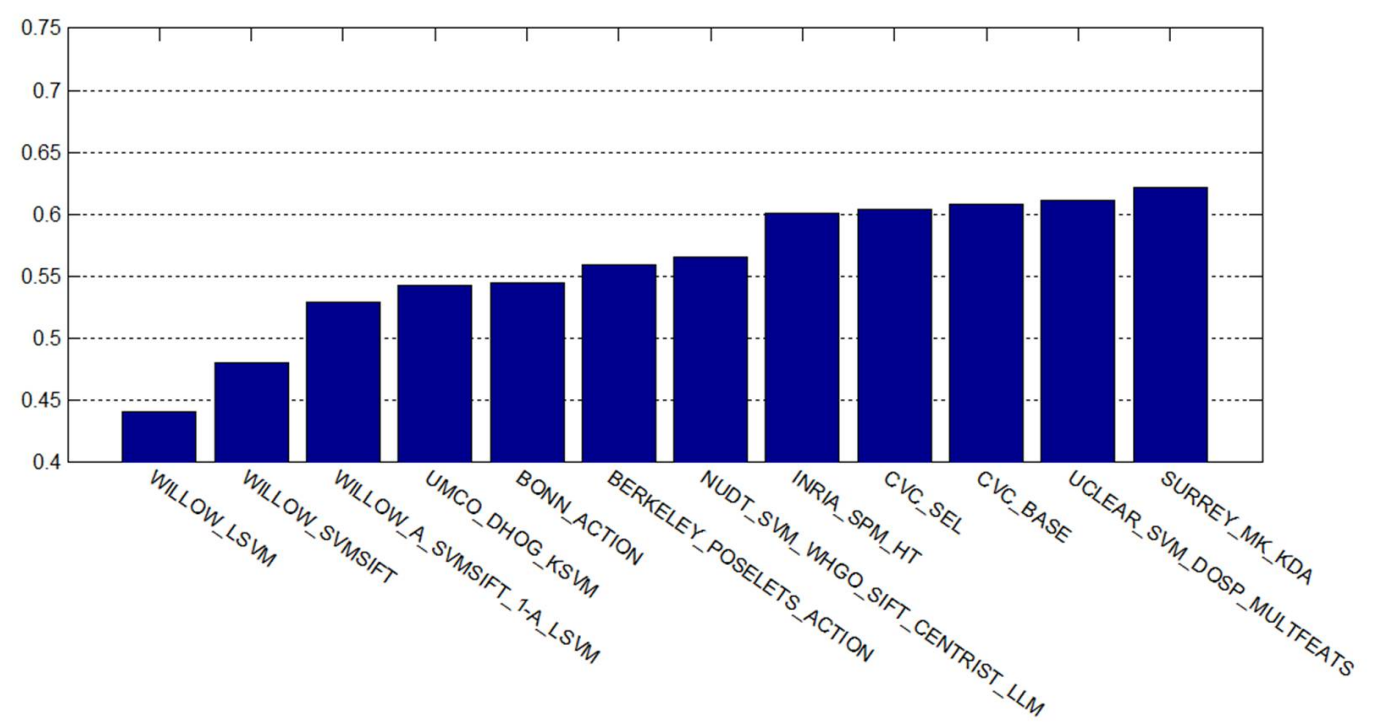

Figure 3.1: Pascal VOC 2010 Action Dataset - Performance comparison of different methods on Pascal VOC 2010 Action Dataset 9 .

research advances, but the improvements are not big, e.g., less than $10 \%$. This indicates that new approaches are still demanding to make more significant progresses. 


\section{4}

\section{Action Learning}

In this chapter, we present our proposed methods, exploring all convenient and possible cues for a single image: the whole image(scene/context), human figure, action objects and human-object interaction. We follow an order of image representation, then action learning and finally showing experiment results. We have demonstrated a state-of-art performance on both Pascal VOC 2010 and 2011 datasets.

\subsection{Image Representation}

In still image-based action recognition, there is no temporal information available, and thus the traditional spatiotemporal features $[31\rfloor$ cannot be applied anymore. Further, in traditional video-based action recognition, the low-level features extracted from spacetime volume can be used directly for action recognition, e.g., the spatiotemporal interest point (STIP) based features. However, in still image-based action recognition, usually the low-level features extracted directly from the whole image cannot work well. Thus previous works seldom use the whole image or scene only for low-level feature extraction and action recognition.

Since only the spatial information is available in single images with cluttered background, researchers have pursued different high-level cues in still images in order to characterize actions better than using low-level features in the whole image. The highlevel cues can be characterized through various low-level features. Then different highlevel cues can be combined to recognize the actions in still images. In this section, we 
will present the high-level cues we used some of which are represented by some low-level features.

\subsubsection{Whole Image}

Generally, scene/context corresponds to the place where an action is being performed such as tennis court and croquet field. Each image is associated with a probability of belonging to one of the scene classes. In most cases, a whole action image is taken as scene/context to be analyzed to extract scene information. Following most other methodologies, we use the whole image to denote scene/context and extract 512dimension GIST feature and 800-dimension dense sampling color DSIFT feature from the whole image for both training and testing images.

The GIST $[30\rfloor$ feature is mainly used to integrate scene or background information. Spatial envelop or called GIST was proposed by Oliva and Torralba in 2001. A set of holistic, spatial properties of the scene can be computed by the GIST method. The GIST is an abstract representation of the scene that spontaneously activates memory representations of scene categories (a city, a mountain, etc.).

The original SIFT algorithm was proposed by David Lowe in 1999 [28], which can detect the interest point locations too. The SIFT feature has been applied in many problems, including object recognition, robotic mapping and navigation, image stitching, 3D modeling, gesture recognition, video tracking, etc. In dense sampling of the SIFT feature, or DSIFT, a regular grid is used to "assign" interest point locations for feature extraction. Given the DSIFT features extracted from many image patches, a clustering is usually executed to obtain a limited number of "keywords" or "codebook", and the histogram can be computed and used as the feature for each image. Color sift is the one of the variant of the DISFT feature.

\subsubsection{Human Body}

Human is the principal part of an action. Human figure centered in the action image with clearly distinct body contour and fixed positioned body parts give clues about what action is being performed. This is why action recognition datasets usually explicitly provide human bounding box or provide a relative center $\lfloor 9\rfloor$ instead of assigning a task of performing human localization before action recognition. We define the provided 
bounding area as the human cue and also extract both 512-dimension GIST and 500dimension color DSIFT feature from the human cues.

\subsubsection{Action Object}

An action object, if exists, provide an important clue to recognize a person's pose, then to indicate the right action one is doing. For most cases, researchers use automatic object localization to label the possible action objects. In this way, due to the occurrence of background object and the insufficient localization methods, it is more difficult to automatically find the right action objects.

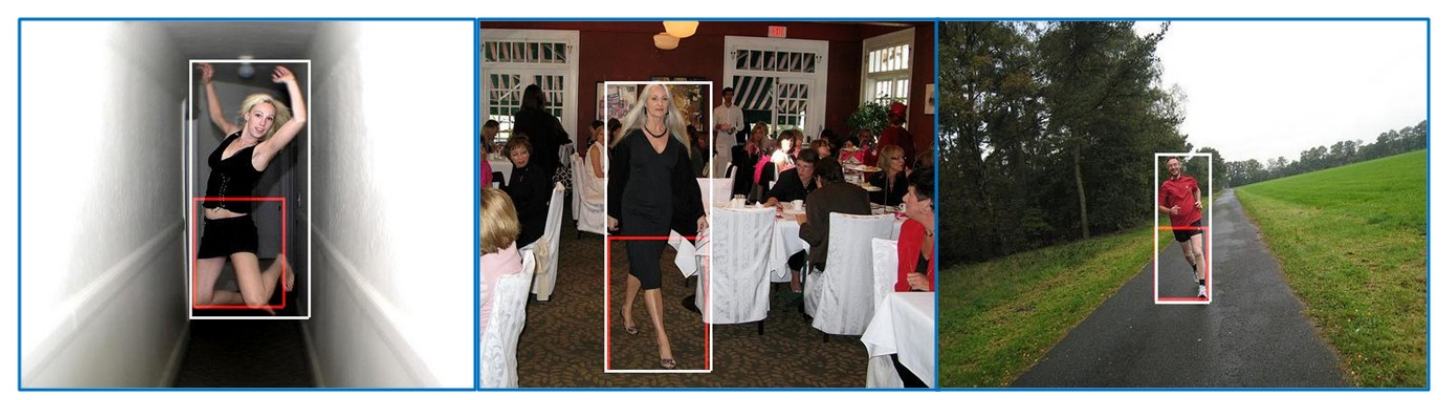

Figure 4.1: Jumping, walking and running.

Inspired by the fact that no matter in a training image or a test image, the human bounding box is usually given, it is suggested that object bounding box can be used in the same way. We assume an object bounding box is much likely to improve efficiency for the recognition of human action. So instead of a weakly supervised way, we manually label the action objects in both training image and test images.

We first detect 100 to 300 windows from around the human box in each image (eg. expand 2 times the box containing larger area from surroundings) by running objectness $[3\rfloor$. For some class like riding horse, 100 windows is enough since usually 100 windows include a lot of large windows that can bound the horse with the human. While in other classes like taking photo, we would like to get more windows since results detected by objectness approach to contain more smaller windows with fine texture such as the camera. In this way, images are first inspected subjectively for what is an object and then followed by human selection for the best window containing the object that promotes the action. 

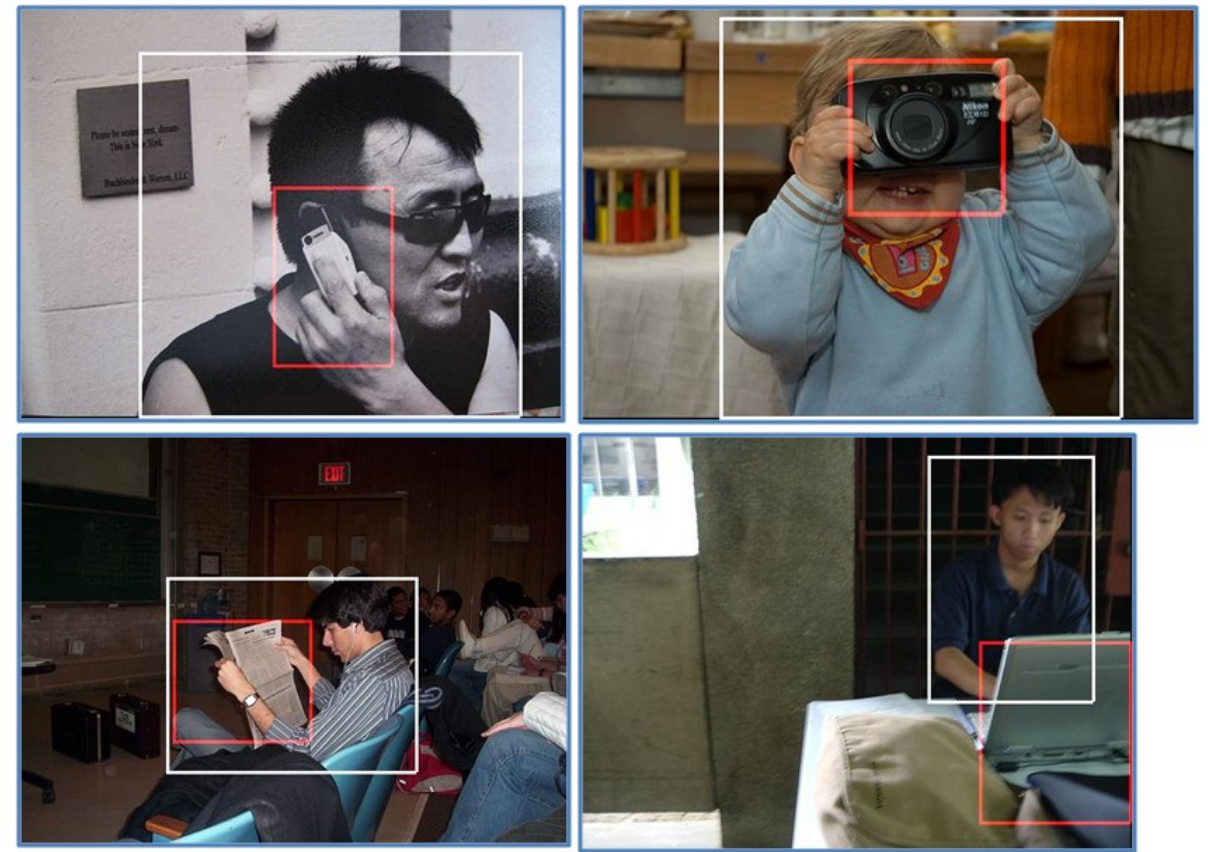

Figure 4.2: Phoning,taking photo, reading and using computer.

Some object bounding box samples are shown in the above figures. In the actions involving no substantial objects such as walking, we use the driving force for an action as the object such human's legs. Then within these bounding boxes, we extracted 500-dimension Color DSIFT feature and the 500-dimension SURF features for representation.

The Speeded Up Robust Features (SURF) $\lfloor 4\rfloor$ feature is a scale- and rotationinvariant interest point detector and descriptor, proposed by Herbert Bay et al. in 2006, partly inspired by the SIFT. It has good performance in tasks such as object recognition or 3D reconstruction. The SURF operator is based on computing the sums of $2 \mathrm{D}$ Haar wavelet responses using the integral images, and thus it can be computed efficiently. For action recognition, Prest et al. $\lfloor 32\rfloor$ showed the use of SURF to extract features from the candidate bounding boxes of action-related objects.

\subsubsection{Human-Object Interaction}

Inspired by $\lfloor 32\rfloor$, several inter-relations between human and action objects are proposed here. Modeling relations including relative scale or location or distance between human 

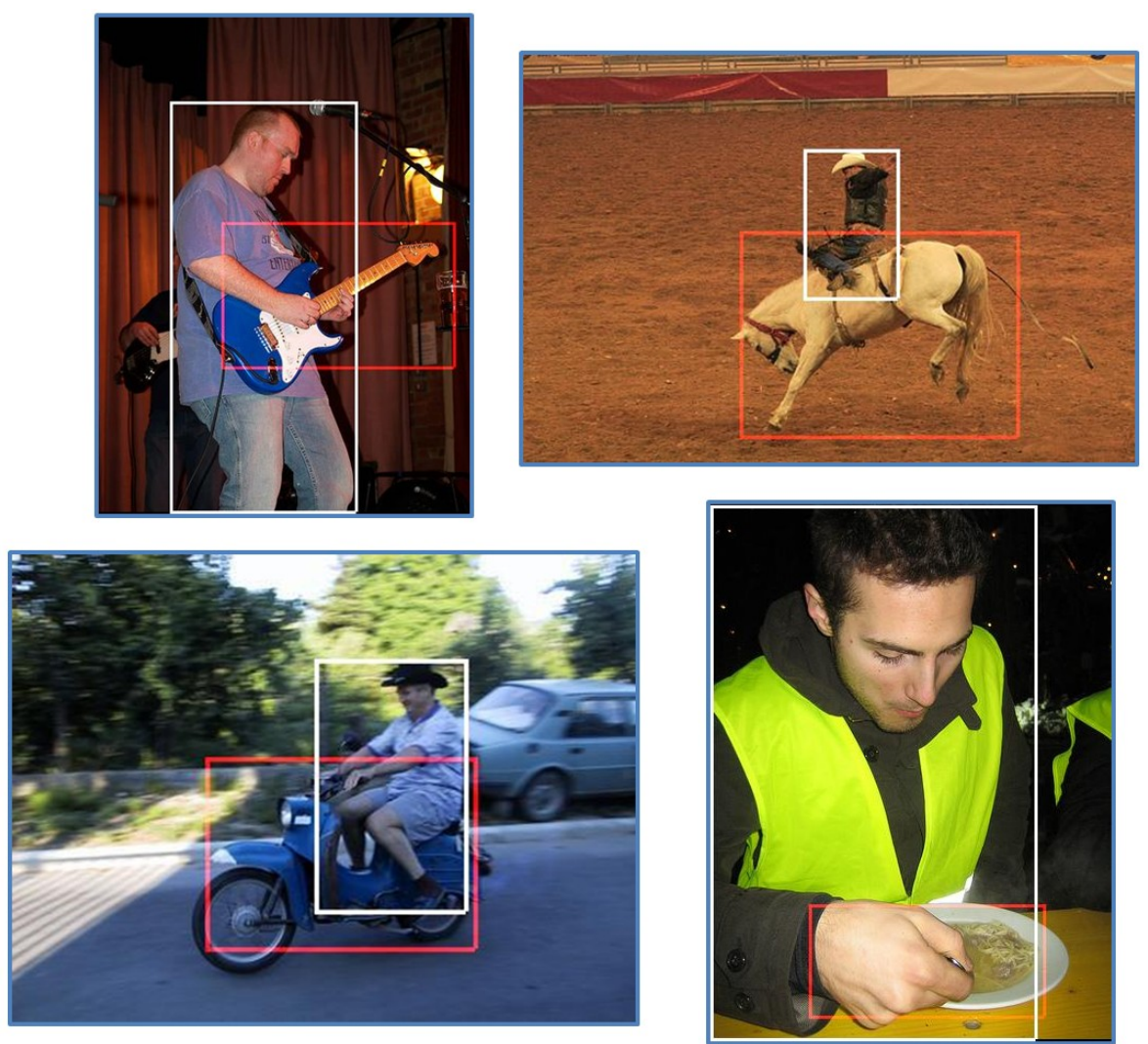

Figure 4.3: Playing instrument,riding horse, riding bike and other actions(eating in the figure).

and object gives an explicit presentation of the interaction in a single image. With prior knowledge in such daily activity or sports, one can easily guess what will happen by seeing how human interacts with the action object, gaining the same effects as from a sequence of images or a video. Here we model 8 kinds of interactions between human and objects, provided the human and object bounding boxes.

\subsubsection{1 relative location}

$$
\begin{gathered}
x=\left(x_{o}-x_{h}\right) / W_{h} \\
y=\left(y_{o}-y_{h}\right) / H_{h} \\
\text { location }=e^{-\frac{x^{2}+y^{2}}{\sigma^{2}}} ;
\end{gathered}
$$


$W_{h}$ and $H_{h}$ are the width and height of the human box. $\left(x_{o}, y_{o}\right)$ and $\left(x_{h}, y_{h}\right)$ are center coordinates of object box and human box.

\subsubsection{2 relative scale}

$$
\text { scale }=e^{-\frac{\operatorname{area}(o) / \operatorname{area}(h)}{\sigma}} ;
$$

\subsubsection{3 relative distance}

$$
\text { distance }=e^{-\frac{\sqrt{x^{2}+y^{2}}}{\sigma}}
$$

$(x, y)$ is relative location from 1.

\subsubsection{4 overlap relation}

$$
\text { overlap }= \begin{cases}\epsilon & \text { ifobj } \cap \text { hum }=\varnothing \\ \frac{\operatorname{area}(o) \cap \operatorname{area}(h)}{\operatorname{area}(h)} & \text { ifobj } \cap \text { hum } \neq \varnothing\end{cases}
$$
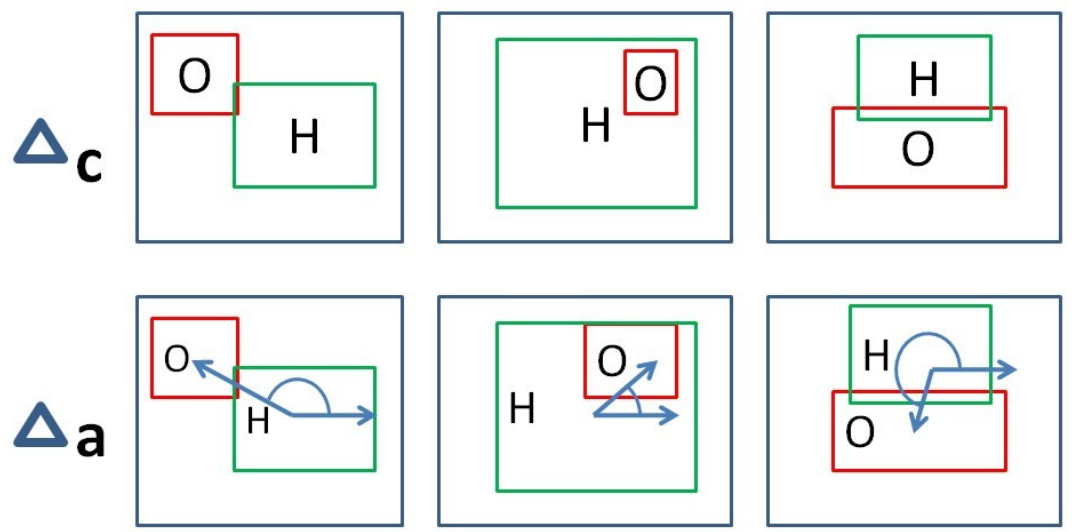

Figure 4.4: contain, angle relations.

\subsubsection{5 containing relation}

$$
\text { contain }= \begin{cases}\epsilon & \text { ifobj } \cap \text { hum }=\varnothing \\ \frac{\operatorname{area}(o) \cap a r e a(h)}{\operatorname{area}(o)} & \text { ifobj } \cap \text { hum } \neq \varnothing\end{cases}
$$




\subsubsection{6 relative angle}

$$
\begin{gathered}
\triangle x=x_{o}-x_{h} \\
\triangle y=y_{o}-y_{h} \\
\theta= \begin{cases}\arctan \frac{\triangle x}{\triangle y} & \text { if } \triangle x>0 \text { and } \triangle y>0 \\
\arctan \frac{\triangle x}{\triangle y}+2 \pi & \text { if } \triangle x>0 \text { and } \triangle y<0 \\
\arctan \frac{\triangle x}{\triangle y}+\pi & \text { if } \triangle x<0\end{cases}
\end{gathered}
$$

$\left(x_{o}, y_{o}\right)$ and $\left(x_{h}, y_{h}\right)$ are center coordinates of object box and human box.
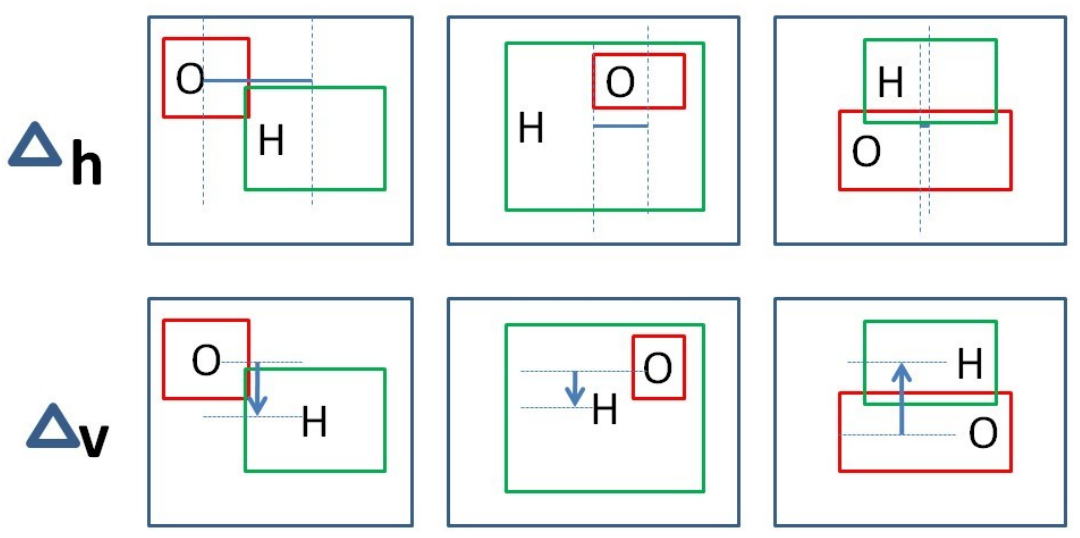

Figure 4.5: horizontal, vertical relations.

\subsubsection{7 horizontal relation}

$$
x=\left|x_{o}-x_{h}\right| / W_{h}
$$

\subsubsection{8 vertical relation}

$$
y=\left(y_{o}-y_{h}\right) / H_{h}
$$

\subsection{Learning}

Using the above image representation which are almost histogram for each image cue, we would like to train classifiers for each single cue or any combination of them. Two kind of machine learning classifiers are chosen: support vector machine or random forest. 


\subsubsection{Support Vector Machine}

Support vector machine(SVM) is first introduced by Cortes and Vapnik [34], this classification method has been widely used in data analysis and pattern recognition. Recently, SVM has been widely used as a classification tool in computer vision, and gets so much popularity. The basic SVM takes a set of input data and predicts, for each given input, which of two possible classes forms the output, making it a non-probabilistic binary linear classifier.

Given a set of training examples, each marked as belonging to one of two categories, an SVM training algorithm builds a model that assigns new examples into one category or the other. An SVM model is a representation of the examples as points in space, mapped so that the examples of the separate categories are divided by a clear gap that is as wide as possible. New examples are then mapped into that same space and predicted to belong to a category based on which side of the gap they fall on.

\subsubsection{Random Forest}

Random forest $(\mathrm{RF})$ is an ensemble learning method for classification (and regression) that operate by constructing a multitude of decision trees at training time and outputting the class that is the mode of the classes output by individual trees. We also try to use a new random forest improved by B. Yao [48], which use a SVM for spitting nodes and make it a stronger classifier.

\subsection{Experiments}

For the first three image cues we have extracted two low-level feature histograms, giving a total of more than 1000 dimension. For human-object interaction, we have 8-dimension feature vector. For each feature vector, we train a random forest and get $\mathrm{N}$ confidence score to indicate the possibility of belonging to $\mathrm{N}$ action classes.

Giving these four probability vectors, we use a weighted linear score-level combination of their confidences. Finally we have $\mathrm{N}$ dimension vector, indicating the ultimate probability for each class. The class with the highest confidence define its label.

We perform our evaluation on both Pascal VOC 2010 and 2011 datasets. Together with the human-object relation modeling, our whole model achieve the highest mAP based on the Pascal VOC action recognition competition evaluation. 
Table 4.1: Pascal VOC 2010

\begin{tabular}{|l|l|l|l|l|l|l|l|l|l|l|}
\hline & Phone & Inst. & Read & Bike & Horse & Run & Photo & Comp. Walk & Avg. \\
\hline surrey mk kda_9. & 52.60 & 53.50 & 35.90 & 81.00 & 89.30 & 86.50 & 32.80 & 59.20 & 68.60 & 62.16 \\
\hline conf score 9. & 49.50 & 56.60 & 31.40 & 82.30 & 89.30 & 87.00 & 36.10 & 67.70 & 73.00 & 63.66 \\
\hline sparse bases_9. & 42.80 & 60.80 & 41.50 & 80.20 & 90.60 & 87.80 & 41.40 & 66.10 & 74.40 & 65.07 \\
\hline stanford rf[48. & 45.00 & 57.40 & 41.50 & 81.80 & 90.50 & 89.50 & 37.90 & 65.00 & 72.70 & 64.59 \\
\hline weakly supervised[32_. & 55.00 & 81.00 & 69.00 & 71.00 & 90.00 & 59.00 & 36.00 & 50.00 & 44.00 & 61.67 \\
\hline phraselets_8. & 47.80 & - & - & 82.20 & 87.00 & 82.80 & 33.70 & 54.50 & 66.90 & - \\
\hline our model & 59.04 & 63.78 & 40.83 & 92.18 & 97.73 & 92.47 & 64.85 & 77.96 & 84.10 & 74.77 \\
\hline
\end{tabular}

Table 4.2: Pascal VOC 2011

\begin{tabular}{|l|l|l|l|l|l|l|l|l|l|l|l|}
\hline & Jump & Phone & Inst. & Read & Bike & Horse & Run & Photo & Comp. Walk & Avg. \\
\hline nudt 9. & 65.90 & 41.50 & 57.40 & 34.70 & 88.80 & 90.20 & 87.90 & 25.70 & 54.50 & 59.50 & 60.61 \\
\hline stanford 46. & 66.70 & 41.10 & 60.80 & 42.20 & 90.50 & 92.20 & 86.20 & 28.80 & 63.50 & 64.20 & 63.62 \\
\hline caenlear 4. & 71.60 & 50.70 & 77.50 & 37.80 & 86.50 & 89.50 & 83.80 & 25.10 & 58.90 & 59.20 & 64.06 \\
\hline Stanford RF 48. & 66.00 & 41.00 & 60.00 & 41.50 & 90.00 & 92.10 & 86.60 & 28.80 & 62.00 & 65.90 & 63.39 \\
\hline 2.5D Graph 44. & 72.40 & 48.30 & 77.70 & 43.20 & 89.00 & 90.00 & 86.80 & 27.90 & 60.50 & 62.10 & 65.79 \\
\hline our model & 73.58 & 46.28 & 64.58 & 45.12 & 92.55 & 96.20 & 87.74 & 30.80 & 71.83 & 69.87 & 67.86 \\
\hline
\end{tabular}

\subsubsection{Overall Performance}

We could see from the table: compared with other competitive methodologies, our model still achieve the highest result on both VOC 2010 and VOC 2011 by at least around $10 \%$ by average on 2010 and at least $2 \%$ by average on 2011 . Also for each class, we excel others a lot more.

\subsubsection{Single Action Image Cues}

For each single image cue, we would like to know how much they contribute to the whole model, Then we construct the following experiments. From both tables, we could see for some classes, human figure or action objects or human-object interaction have more determining effect than other cues due to the action property. But only with the whole image, considering all visual clue from the whole image does not help find the critical clue to decide which action it is. 
Table 4.3: Pascal VOC 2010 Single Cue

\begin{tabular}{|l|c|c|c|c|c|c|c|c|c|c|}
\hline & Phone & Inst. & Read & Bike & Horse & Run & Photo & Comp. Walk & Avg. \\
\hline whole image & 29.88 & 50.76 & 27.33 & 27.75 & 52.03 & 46.32 & 24.58 & 38.82 & 29.89 & 36.37 \\
\hline human only & 24.53 & 29.05 & 32.75 & 34.50 & 57.59 & 72.11 & 24.47 & 32.13 & 51.46 & 39.84 \\
\hline object only & 40.52 & 37.53 & 29.21 & 40.74 & 72.45 & 62.14 & 44.26 & 57.23 & 58.23 & 49.15 \\
\hline inte. only & 36.51 & 33.48 & 21.34 & 47.08 & 72.88 & 52.40 & 50.69 & 50.05 & 49.39 & 45.98 \\
\hline
\end{tabular}

Table 4.4: Pascal VOC 2011 Single Cue

\begin{tabular}{|l|l|l|l|l|l|l|l|l|l|l|l|}
\hline & Jump & Phone & Inst. & Read & Bike & Horse & Run & Photo & Comp. Walk & Avg. \\
\hline whole image & 30.30 & 32.56 & 50.78 & 24.36 & 32.99 & 33.29 & 40.81 & 12.97 & 34.55 & 23.58 & 31.62 \\
\hline human only & 43.98 & 35.28 & 36.00 & 25.21 & 53.74 & 56.81 & 59.54 & 15.64 & 27.82 & 41.65 & 39.57 \\
\hline object only & 26.78 & 28.04 & 39.82 & 30.67 & 42.61 & 55.35 & 46.02 & 12.08 & 41.61 & 40.30 & 36.33 \\
\hline inte. only & 31.65 & 22.22 & 26.58 & 23.66 & 40.01 & 73.35 & 29.14 & 20.89 & 35.40 & 35.19 & 33.81 \\
\hline
\end{tabular}

\subsubsection{Object Localization}

In this subsection, we would like to perform automatic object localization on test images. We use only labelled object boxes from training images but leave testing images intact, trying to localize action object inferred from training data.

\subsubsection{Object Candidate}

We approach to find objects in a way similar to weakly supervised $[32\rfloor$. They find the best coherent configuration for the human-object relations from between the training images, giving a best object box for each designated human box. And from these training boxes, they learn the most probable object windows fitting each candidate training model. At the end, they have one best object window for each class. Different from the training stages, we would still use our manually label object windows for training images.

We have a general ideas about the preliminary results about the most probably classes or each image by running classifiers on human figure and whole image, based on the assumption that the action class give a greater confidence about the object class in the same image. So we limit our candidate object classes according to top $\mathrm{N}$ classes with highest probabilities for each testing image. An optimal $\mathrm{N}$ is found to be 6 , achieving a high accuracy as $96 \%$. So we decide that for each testing image, objectness 
would be run to get object candidate windows for each of the top 6 possible classes per testing image.

Since human boxes are also given on the testing images, we regard this as a prior knowledge and run objectness in the same way as previous to label object, getting 100 to 300 candidate object windows for different classes, assuming that for each of the 6 classes these object windows include the true action object for that image.

\subsubsection{Candidate Elimination}

Using the interaction relations (usually presented as mean \pm variance)learned from training images, we can eliminate a larger part of these candidate boxes for each candidate class. We decide boxes falling within the range given by the training interactions can remain to be candidate for that class, forming a total of 10 to 30 boxes left.

For the resting boxes, we learn both their spatial and appearance features in a way similar to $\lfloor 32\rfloor$, after we perform a simple merge between these boxes. Compared to the training objects, we can decide a rank for these candidate windows a confidence for their probable class. At the end we could decide that a rank $\mathrm{M}$ object windows are used to be the object boxes. $\mathrm{M}$ is selected to be 1 . In the situation of more than 1 candidate boxes, majority voting is performed to decide the final object label for the object window with the largest probability denoting that label.

\subsubsection{Object Representation}

Using these found objects, we can combine the previous features extracted from whole image, human bounding box $\mathrm{t}$ in the same way in our previous experiments. We can get the below results, which shows that though our object localization is insufficient to get exact label for test image, it is still competitive to maintain relative advantage compared to most other methods. 
Table 4.5: Pascal VOC 2010 - object localiztion

\begin{tabular}{|l|l|l|l|l|l|l|l|l|l|l|}
\hline surrey mk kda_9. & 52.60 & 53.50 & 35.90 & 81.00 & 89.30 & 86.50 & 32.80 & 59.20 & 68.60 & 62.16 \\
\hline conf score 9. & 49.50 & 56.60 & 31.40 & 82.30 & 89.30 & 87.00 & 36.10 & 67.70 & 73.00 & 63.66 \\
\hline sparse bases_9. & 42.80 & 60.80 & 41.50 & 80.20 & 90.60 & 87.80 & 41.40 & 66.10 & 74.40 & 65.07 \\
\hline stanford rf[48_ & 45.00 & 57.40 & 41.50 & 81.80 & 90.50 & 89.50 & 37.90 & 65.00 & 72.70 & 64.59 \\
\hline weakly supervised_32_. & 55.00 & 81.00 & 69.00 & 71.00 & 90.00 & 59.00 & 36.00 & 50.00 & 44.00 & 61.67 \\
\hline phraselets_8. & 47.80 & - & - & 82.20 & 87.00 & 82.80 & 33.70 & 54.50 & 66.90 & - \\
\hline our model & 59.04 & 63.78 & 40.83 & 92.18 & 97.73 & 92.47 & 64.85 & 77.96 & 84.10 & 74.77 \\
\hline our model(auto) & 50.97 & 58.57 & 35.23 & 88.69 & 94.91 & 90.72 & 39.95 & 70.81 & 79.4 .7 & 67.7 \\
\hline
\end{tabular}

Table 4.6: Pascal VOC 2011 - object localiztion

\begin{tabular}{|l|l|l|l|l|l|l|l|l|l|l|l|}
\hline & Jump & Phone & Inst. & Read & Bike & Horse & Run & Photo & Comp. Walk & Avg. \\
\hline nudt 9. & 65.90 & 41.50 & 57.40 & 34.70 & 88.80 & 90.20 & 87.90 & 25.70 & 54.50 & 59.50 & 60.61 \\
\hline stanford 46. & 66.70 & 41.10 & 60.80 & 42.20 & 90.50 & 92.20 & 86.20 & 28.80 & 63.50 & 64.20 & 63.62 \\
\hline caenlear 9. & 71.60 & 50.70 & 77.50 & 37.80 & 86.50 & 89.50 & 83.80 & 25.10 & 58.90 & 59.20 & 64.06 \\
\hline Stanford RF 48. & 66.00 & 41.00 & 60.00 & 41.50 & 90.00 & 92.10 & 86.60 & 28.80 & 62.00 & 65.90 & 63.39 \\
\hline 2.5D Graph 44_ & 72.40 & 48.30 & 77.70 & 43.20 & 89.00 & 90.00 & 86.80 & 27.90 & 60.50 & 62.10 & 65.79 \\
\hline our model & 73.58 & 46.28 & 64.58 & 45.12 & 92.55 & 96.20 & 87.74 & 30.80 & 71.83 & 69.87 & 67.86 \\
\hline our model(auto) & 65.72 & 45.32 & 62.34 & 42.12 & 89.89 & 93.26 & 86.7 & 27 & 64.89 & 66.09 & 64.33 \\
\hline
\end{tabular}




\section{5}

\section{Discussion}

Based on our overview and presentation in previous sections, one can see that significant progresses have been made in still image-based action recognition. However, the field of research is still in its early stage. Deeper studies of methodologies are expected to make a breakthrough in the area. Here we present our own views and thoughts, and hope these can inspire new research efforts.

\subsection{Discussion}

(1) How many action classes can be collected for still image-based action recognition?

In Chapter 3, we introduced a list of databases for action recognition in still images. Most of the databases contain about 10 action classes or less, while two databases have 40 or up to 89 action classes. So the question is, how many action classes can be collected? What is the maximum number of action classes in reality? We believe that 89 is not the maximum number of action classes. As a multi-class classification problem, the number of classes does matter in evaluating different methods. Suppose we know the maximum number of action classes, denoted by $M$, then a unique benchmark dataset might be built, and all future developed methods can use the same database for validation and comparisons. Some related issues include: Among the $M$ total classes, which action classes are the most difficult to separate from others? Does there exist any "easy" or "hard" actions to recognize, among the $M$ classes in total?

(2) How many cues can be found for still image-based action recognition? In Chapter 2 , we presented various high-level cues for action analysis in still images, including 
human body, body parts, object, human object interaction, and the scene or context. A question is, can we find some new cues to enhance action recognition? If yes, what are those new cues? How to represent them? By addressing these questions, one may develop new approaches to improve the action recognition performance.

(3) What features are appropriate for image-based action recognition? In previous chapters, we presented various low-level features for high-level cues representation and action recognition. Almost all those features were originally developed for other computer vision problems. A question may be asked: Can we have some "special" features that are unique for action representation in still images? There are some existing approaches to learn mid-level features. However, new features are expected to target the action patterns specifically, and develop a better representation than the current features.

(4) How to combine action recognition with other research problems? In traditional video-based action recognition, the problem is often taken as an independent one. While in still image-based action recognition, it usually needs the cues about human, objects, human body pose, human object interaction, and so on. The research progresses in related areas, as we discussed in previous chapters, will definitely help to improve still image-based action recognition. For instance, the human bounding boxes are currently provided in many action databases based on manual labelling. It will be nice if an automated human detection can achieve a high accuracy, e.g., above 95\%, in action images. On the other hand, action recognition in still images might help to enrich solving other problems. For example, automated action analysis in still images can "tag" online images with the performed actions for image search or retrieval. This will make the action recognition more interesting, and may bring more attention to researchers in image or multimedia retrieval community.

(5) How to solve the occlusion problem in action recognition? Many high-level cues and low-level features are usually needed for action analysis. Sometimes, occlusions could be serious. For instance, the human body may occlude objects (fully or partially) or be occluded partially by the objects, or human body parts may self-occlude each other. The occlusions may cause it difficult to extract the related high-level cues or lowlevel features. There are some approaches, e.g., [8, using a visibility flag to indicate a particular body part being occluded or not. However, further efforts are needed to deal 
with the occlusions, making the computational approach insensitive to full or partial occlusions.

(6) How to do action learning in the small sample case? As we discussed in Chapter 3 , some of the existing databases have more image examples in each class, while some others have less. The Pascal VOC databases are continuously increasing the number of image examples for each class in each year. So some questions can be raised: How many examples are needed to learn the action classes? Is there a minimum number of training examples for each action class? Do we have enough training examples to represent all possible variations for each action class? If the number of training examples is too small, compared to all possible variations, we have the problem of learning in the small sample case. How to develop robust methods to learn actions with small samples?

(7) Which actions are appropriate to use videos and which to use still images? There are many action databases, either videos or still images. If we look at the specific actions in these two types of action databases, we can find that some actions appear in both videos and still images, such as walking, running, etc. There are also actions that appear in still image databases but not in videos, or vice versa. We may ask a question: Which actions are appropriate to use videos for representation, and which are proper to use still images? If an action can be characterized completely by a still image, probably there is no need to capture and store in a video. Even stored in a video, one may use a small number of image frames for analysis of that action which is appropriate to use still images. On the other hand, if some actions need to use video data for a better representation, we may not have a good performance for those actions using still images, and we know why. Further, researchers will not waste time to develop new algorithms to improve the performance for those actions appeared in still images.

(8) Are discriminative methods good enough to separate action classes? Or are generative models better for certain actions? For action learning, there are both general and discriminative approaches. We may ask questions: Which learning method is better for action recognition on standard databases: discriminative or generative? Does discriminative learning perform better than the generative for some actions, but worse for some others? Based on these studies, one may find the appropriate learning methods for action recognition in still images. 


\subsection{Concluding Remarks}

In our work, we present a new problem of recognizing human action and our methodologies to solve this problem. We have also conducted a comprehensive survey of existing approaches to still image-based action recognition. We have introduced different approaches based on a categorization of them with high-level cues and low-level features. Different action learning methods have been discussed too. Various action databases are grouped and summarized with specific details. We have also presented some research topics that are related to action analysis in still images, and given some thoughts for future research. As a relatively new area, the research on still image-based action recognition is in the early stage. The recognition accuracies are not high based on examining the results on several often-used databases.

We present our experiments using different low-level features and high-level image cues, Moreover, we manually label the action objects from both training and testing images, inspired by labelling of human bounding boxes given along with the datasets. We have demonstrated the effects of using random forest combined with score-level fusion on the labelled data, providing an upper bound of the action recognition with objects. Instead of labels on the testing images, object localization is performed.

In our future research, our plan is to enhance our object localization methodology to improve the performance of our automatic object labelling algorithm. 


\section{Bibliography}

[1] J. Aggarwal and Q. Cai. Human motion analysis: A review. Computer Vision and Image Understanding, 73:428-440, 1999.

[2] J. Aggarwal and M. Ryoo. Human activity analysis: A review. ACM Computing Surveys (CSUR), 43(3):16, 2011.

[3] B. Alexe, T. Deselaers, and V. Ferrari. What is an object? In Computer Vision and Pattern Recognition, IEEE Conference on, pages 73-80. IEEE, june 2010.

[4] H. Bay, T. Tuytelaars, and L. V. Gool. Surf: Speeded up robust features. In Computer Vision, European Conference on, pages 404-417. Springer Berlin / Heidelberg, 2006.

[5] C. Cedras and M. Shah. Motion-based recognition a survey. Image and Vision Computing, 13(2):129-155, 1995.

[6] N. Dalal and B. Triggs. Histograms of oriented gradients for human detection. In Computer Vision and Pattern Recognition, IEEE Conference on, pages 886-893. IEEE, june 2005.

[7] V. Delaitre, I. Laptev, and J. Sivic. Recognizing human actions in still images: a study of bag-of-features and part-based representations. In Proceedings of the British Machine Vision Conference, page 7. BMVA, 2010.

[8] C. Desai and D. Ramanan. Detecting actions, poses, and objects with relational phraselets. In Computer Vision Workshops and Demonstrations, European Conference on, pages 158172. Springer-Verlag, 2012.

[9] M. Everingham, L. Van Gool, C. K. I. Williams, J. Winn, and A. Zisserman. The pascal visual object classes (voc) challenge. International Journal of Computer Vision, 88(2):303338 , June 2010.

[10] B. Fasel and J. Luettin. Automatic facial expression analysis: a survey. Pattern Recognition, 36(1):259-275, 2003.

[11] L. Fei-Fei, R. Fergus, and P. Perona. Learning generative visual models from few training examples: An incremental bayesian approach tested on 101 object categories. Computer Vision and Image Understanding, 106(1):59-70, 2007.

[12] D. Gavrila. The visual analysis of human movement: A survey. Computer vision and image understanding, 73(1):82-98, 1999.

[13] L. Gorelick, M. Blank, E. Shechtman, M. Irani, and R. Basri. Actions as space-time shapes. In $I C C V$, pages 1395-1402, 2005.

[14] A. Gupta, A. Kembhavi, and L. Davis. Observing human-object interactions: Using spatial and functional compatibility for recognition. Pattern Analysis and Machine Intelligence, 
IEEE Transactions on, 31(10):1775-1789, oct. 2009.

[15] N. Ikizler, R. Cinbis, S. Pehlivan, and P. Duygulu. Recognizing actions from still images. In Pattern Recognition, International Conference on, pages 1-4. IEEE, dec. 2008.

[16] N. Ikizler-Cinbis, R. G. Cinbis, and S. Sclaroff. Learning actions from the Web. In Computer Vision, IEEE International Conference on, pages 995-1002. IEEE, 2009.

[17] N. Ikizler-Cinbis and S. Sclaroff. Web-based classifiers for human action recognition. Multimedia, IEEE Transactions on, 14(4):1031-1045, 2012.

[18] X. Ji and H. Liu. Advances in view-invariant human motion analysis: A review. Systems, Man, and Cybernetics, Part C: Applications and Reviews, IEEE Transactions on, 40(1):1324, 2010.

[19] F. S. Khan, M. A. Rao, J. van de Weijer, A. D. Bagdanov, A. Lopez, and M. Felsberg. Coloring action recognititon in still images. International Journal of Computer Vision (IJCV), pages 1-17, 2013.

[20] P. Koniusz and K. Mikolajczyk. Soft assignment of visual words as linear coordinate coding and optimisation of its reconstruction error. In Image Processing, IEEE International Conference on, pages 2413-2416. IEEE, sept. 2011.

[21] P. Koniusz and K. Mikolajczyk. Spatial coordinate coding to reduce histogram representations, dominant angle and colour pyramid match. In Image Processing, IEEE International Conference on, pages 661-664. IEEE, sept. 2011.

[22] V. Kruger, D. Kragic, A. Ude, and C. Geib. The meaning of action: a review on action recognition and mapping. Advanced Robotics, 21(13):1473-1501, 2007.

[23] M. P. Kumar, B. Packer, and D. Koller. Modeling latent variable uncertainty for loss-based learning. In Proceedings of International Conference on Machine Learning, pages 465-472, New York, NY, USA, July 2012. Omnipress.

[24] S. Lazebnik, C. Schmid, and J. Ponce. Beyond bags of features: Spatial pyramid matching for recognizing natural scene categories. In Computer Vision and Pattern Recognition, IEEE Conference on, volume 2, pages 2169-2178. IEEE, 2006.

[25] D. T. Le, R. Bernardi, and J. Uijlings. Exploiting language models to recognize unseen actions. In Proceedings of the 3rd ACM conference on International conference on multimedia retrieval, pages 231-238. ACM, 2013.

[26] L.-J. Li and L. Fei-Fei. What, where and who? classifying events by scene and object recognition. In Computer Vision, IEEE International Conference on, pages 1-8. IEEE, oct. 2007.

[27] P. Li, J. Ma, and S. Gao. Actions in still web images: Visualization, detection and retrieval. In Web-Age Information Management: 12th International Conference, volume 6897, page 302. Springer, 2011.

[28] D. Lowe. Object recognition from local scale-invariant features. In Computer Vision, IEEE International Conference on, volume 2, pages 1150-1157. IEEE, 1999.

[29] S. Maji, L. Bourdev, and J. Malik. Action recognition from a distributed representation of pose and appearance. In Computer Vision and Pattern Recognition, IEEE Conference on, pages 3177-3184. IEEE, june 2011.

[30] A. Oliva and A. Torralba. Modeling the shape of the scene: A holistic representation of 
the spatial envelope. International Journal of Computer Vision, 42:145-175, 2001.

[31] R. Poppe. A survey on vision-based human action recognition. Image and Vision Computing, 28:976-990, 2010.

[32] A. Prest, C. Schmid, and V. Ferrari. Weakly supervised learning of interactions between humans and objects. Pattern Analysis and Machine Intelligence, IEEE Transactions on, 34(3):601-614, march 2012.

[33] K. Raja, I. Laptev, P. Pérez, and L. Oisel. Joint pose estimation and action recognition in image graphs. In Image Processing, IEEE International Conference on, pages 25-28. IEEE, 2011.

[34] B. Schölkopf and A. J. Smola. Learning with kernels: Support vector machines, regularization, optimization, and beyond. MIT press, 2001.

[35] C. Schuldt, I. Laptev, and B. Caputo. Recognizing human actions: a local svm approach. In Pattern Recognition, IEEE International Conference on, volume 3, pages 32-36. IEEE, aug. 2004.

[36] G. Sharma, F. Jurie, and C. Schmid. Discriminative spatial saliency for image classification. In Computer Vision and Pattern Recognition, IEEE Conference on, pages 3506-3513. IEEE, 2012.

[37] C. Thurau and V. Hlavac. Pose primitive based human action recognition in videos or still images. In Computer Vision and Pattern Recognition, IEEE Conference on, pages 1-8. IEEE, june 2008.

[38] P. Turaga, R. Chellappa, V. Subrahmanian, and O. Udrea. Machine recognition of human activities: A survey. Circuits and Systems for Video Technology, IEEE Transactions on, 18(11):1473-1488, 2008.

[39] Y. Wang, H. Jiang, M. Drew, Z.-N. Li, and G. Mori. Unsupervised Discovery of Action Classes. In Computer Vision and Pattern Recognition, IEEE Conference on, pages 16541661. IEEE, 2006.

[40] P. Welinder, S. Branson, T. Mita, C. Wah, F. Schroff, S. Belongie, and P. Perona. Caltechucsd birds 200. Technical Report CNS-TR-201, Caltech, 2010.

[41] W. Yang, Y. Wang, and G. Mori. Recognizing human actions from still images with latent poses. In Computer Vision and Pattern Recognition, IEEE Conference on, pages 2030-2037. IEEE, june 2010.

[42] B. Yao and L. Fei-Fei. Grouplet: A structured image representation for recognizing human and object interactions. In Computer Vision and Pattern Recognition, IEEE Conference on, pages 9-16, San Francisco, USA, June 2010. IEEE.

[43] B. Yao and L. Fei-Fei. Modeling mutual context of object and human pose in human-object interaction activities. In Computer Vision and Pattern Recognition, IEEE Conference on, pages 17-24. IEEE, june 2010.

[44] B. Yao and L. Fei-Fei. Action recognition with exemplar based $2.5 \mathrm{~d}$ graph matching. In Computer Vision Workshops and Demonstrations, European Conference on, pages 173186. Springer-Verlag, 2012.

[45] B. Yao and L. Fei-Fei. Recognizing human-object interactions in still images by modeling the mutual context of objects and human poses. IEEE Transactions on Pattern Analysis 
and Machine Intelligence, 99(PrePrints):1691-1703, 2012.

[46] B. Yao, X. Jiang, A. Khosla, A. Lin, L. Guibas, and L. Fei-Fei. Human action recognition by learning bases of action attributes and parts. In Computer Vision, IEEE International Conference on, pages 1331-1338. IEEE, nov. 2011.

[47] B. Yao, A. Khosla, and L. Fei-Fei. Classifying actions and measuring action similarity by modeling the mutual context of objects and human poses. In International Conference on Machine Learning, page D3, Bellevue, USA, June 2011. Omnipress.

[48] B. Yao, A. Khosla, and L. Fei-Fei. Combining randomization and discrimination for finegrained image categorization. In Computer Vision and Pattern Recognition, IEEE Conference on, pages 1577-1584. IEEE, june 2011.

[49] Z. Zeng, M. Pantic, G. I. Roisman, and T. S. Huang. A survey of affect recognition methods: audio, visual, and spontaneous expressions. IEEE Trans. Pattern Anal. Mach. Intell., 31(1):39-58, 2009. 